JOURNAL OF THE

AMERICAN MATHEMATICAL SOCIETY

Volume 25, Number 4, October 2012, Pages 1091-1117

S 0894-0347(2012)00739-5

Article electronically published on April 12, 2012

\title{
BRAUER-SIEGEL FOR ARITHMETIC TORI AND LOWER BOUNDS FOR GALOIS ORBITS OF SPECIAL POINTS
}

\author{
JACOB TSIMERMAN
}

\section{INTRODUCTION}

Let $A_{g, 1}$ be the coarse moduli space of principally polarized abelian varieties. Recall that an abelian variety $B$ of dimension $g$ is said to be CM if its endomorphism algebra $\operatorname{End}(B) \otimes_{\mathbb{Z}} \mathbb{Q}$ contains a semi-simple, commutative algebra $R$ over $\mathbb{Q}$ with $[R: \mathbb{Q}]=2 g$. This paper is motivated by the following conjecture, first suggested by Edixhoven in [4]:

Conjecture 1.1. Fix an integer $g$. Let $B$ be a g-dimensional $C M$ principally polarized abelian variety, and let $x$ be the corresponding point in $A_{g, 1}(\overline{\mathbb{Q}})$. Finally, let $Z(\operatorname{End}(B))$ denote the center of the endomorphism ring of $B$. Then there exists a constant $\delta_{g}>0$ so that

$$
|\operatorname{Gal}(\overline{\mathbb{Q}} / \mathbb{Q}) \cdot x| \gg_{g} \operatorname{Disc}(Z(\operatorname{End}(B)))^{\delta_{g}} .
$$

Edixhoven established the desired lower bound for Hilbert modular surfaces in 3.

Recently, Pila 11 gave an unconditional proof of the André-Oort conjecture for an arbitrary product of modular curves. A positive answer to Conjecture 1.1 would be one of the main ingredients in generalizing Pila's recent work to arbitrary Shimura varieties. Our main theorem is as follows:

Theorem 1.1. For $g \leq 6$, Conjecture 1.1 holds. If one assumes the Generalized Riemann Hypothesis for CM fields, then Conjecture 1.1 holds for all $g \in \mathbb{N}$.

We mention that using similar methods, Ullmo and Yafaev independently settled the case of $g=2,3$ [16]. Following Chai and Oort, we define a Weyl CM point to be a CM point $x$ corresponding to an abelian variety $B$ whose endomorphism ring $\operatorname{End}(B)$ is an order in a Weyl CM field. Since for $g=1$ all CM points are Weyl $\mathrm{CM}$ points, the following theorem can be seen as a generalization of Brauer-Siegel's theorem to all $g$ :

Theorem 1.2. Conjecture 1.1 holds for all $g \in \mathbb{N}$ if one restricts to Weyl CM points $x$. Moreover, suppose $x$ corresponds to an abelian variety $B$ whose endomorphism ring $\operatorname{End}(B)$ is the ring of integers in a Weyl $C M$ field $K$ with totally real subfield F. Then

$$
|\operatorname{Gal}(\overline{\mathbb{Q}} / \mathbb{Q}) \cdot x|=\left(\frac{\operatorname{Disc} K}{\operatorname{Disc} F}\right)^{\frac{1}{2}+o_{g}(1)} .
$$

Received by the editors May 29, 2011 and, in revised form, February 27, 2012, and March 23, 2012.

2010 Mathematics Subject Classification. Primary 11G15.

(C) 2012 American Mathematical Society Reverts to public domain 28 years from publication 1091 
For $g=1$, Conjecture 1.1 is answered by the Brauer-Siegel theorem for class groups of imaginary quadratic fields. For higher $g$, however, the question isn't just about understanding the sizes of class groups, but more about the sizes of images of morphisms between class groups. Thus, for higher $g$ instead of class groups of number fields, it turns out that we are forced to deal with class groups of arithmetic tori over $\mathbb{Q}$. To understand the size of the class groups of tori, we prove the following theorem in section 4 :

Theorem 1.3 (Brauer-Siegel Theorem for tori). Let $T$ be a torus of dimension $d$ with minimal splitting field $K$, and $[K: \mathbb{Q}]=n$. Let $h_{T}, R_{T}$ be the class number and regulator of $T$ respectively. Also, let $\rho$ be the associated integral representation of $\mathrm{Gal}(\overline{\mathbb{Q}} / \mathbb{Q})$ and $\rho_{\mathbb{Q}}$ the induced rational representation. Let $f_{\rho}$ be the norm of the Artin conductor corresponding to $\rho_{\mathbb{Q}}$. Then we have

$$
\begin{aligned}
\frac{1}{2} \ln \left(h_{T} R_{T}\right) & =\frac{1}{2} \ln f_{\rho}+o_{n, d}\left(\ln D_{K}\right) \\
& =\left(\frac{1}{2}+o_{n, d}(1)\right) \ln f_{\rho} .
\end{aligned}
$$

Note that all the relevant tori for the study of CM points are compact at infinity, and thus have no regulator. In this case, Theorem 4.7 tells us precisely how large the class group is.

The general setting we study in section 5 is the following: We are given tori $T$ and $S$ over $\mathbb{Q}$, and a map

$$
\phi: T \rightarrow S
$$

between them. This induces a map of class groups

$$
\tilde{\phi}: C l(T) \rightarrow C l(S)
$$

and our goal is to understand the size of the image of $\tilde{\phi}$, or more precisely to get a lower bound for it. One way to do this is to try and bound torsion in class groups of tori: suppose we knew that $\phi$ had an inverse up to isogeny, so that there is some map $\psi: S \rightarrow T$ with $\phi \circ \psi$ the $\times n$ map on $T$. Then it follows from this that the image of $\tilde{\phi}$ is at least the image of the $\times n$ map on $C l(S)$, and so it has size at least $|C l(S)| /|C l(S)[n]|$. Now if we could just give a good bound for $|C l(S)[n]|$ we would be done. Unfortunately, bounding torsion in class groups is a very hard problem in general. We take a more precise approach in section 5 , which describes the size of the cokernel of $\tilde{\phi}$ in terms of the cokernel of the map on characters $X(S) \rightarrow X(T)$ viewed as Galois representations. A nice corollary of this approach is that it allows us to derive new transfer principles between torsion in class groups of number fields, as is explained in section 6 .

The structure of this paper is as follows. In sections 2 and 3 we give some background about arithmetic tori, and recall Shyr's formula, which is the analogue for arithmetic tori of Dirichlet's class number formula for number fields. In section 4 we use Shyr's formula to prove the Brauer-Siegel Theorem for tori, Theorem 4.7. Section 5 is the core of the paper, and it proves a structure theorem for maps between class groups of tori. In section 6 we explain how one can use the results in Section 5 to derive transfer principles for torsion in ideal class groups of number fields, and we give a new application relating 2-torsion in quartic fields to 2-torsion in their cubic resolvents. Finally, section 7 gives the promised applications to lower bounds of Galois orbits of CM abelian varieties. 


\section{Preliminaries}

A torus of dimension $d$ over a field $k$ is an algebraic group $T$ over $k$ such that $T_{\bar{k}}$ is isomorphic to a power of the multiplicative algebraic group $\mathbb{G}_{m}$ so that $T_{\bar{k}} \approx \mathbb{G}_{m / \bar{k}}^{d}$.

Given a torus $T$ of dimension $d$, we define its character group $X(T)$ to be $\operatorname{Hom}_{\bar{k}}\left(T, \mathbb{G}_{m}\right)$, and its cocharacter group $X(T)^{*}$ to be $\operatorname{Hom}_{\bar{k}}\left(\mathbb{G}_{m}, T\right)$. Note that $X(T)$ and $X(T)^{*}$ are free abelian groups on $d$ generators. $X(T)$ also acquires an action of the absolute Galois group $G_{k}:=\operatorname{Gal}(\bar{k} / k)$ via $\chi^{\sigma}(t)=\chi\left(t^{\sigma^{-1}}\right)^{\sigma}$, and similiarly for $X(T)^{*}$. The functor sending $T$ to $X(T)$ (resp. $\left.X(T)^{*}\right)$ is a contravariant (resp. covariant) category equivalence between tori and torsion-free, finitely generated $G_{k}$-modules.

We say that a torus $T$ splits over $K$ if it is isomorphic to a power of $\mathbb{G}_{m}$ over $K$, or equivalently that $X(T)$ is a trivial module for the subgroup $\operatorname{Gal}(\bar{k} / K)$. We define $C(K / k)$ to be the set of all tori over $k$ which are split over $K$. Note that there is a minimal extension $K$ of $k$ over which $T$ becomes split, and $K$ is Galois over $k$. For each extension $L$ of $k$, we define $X(T)_{L}$ to be the subgroup of $X(T)$ fixed by $\operatorname{Gal}(\bar{k} /(L \cap \bar{k}))$. If $v$ is a place of $k$, we sometimes write $X(T)_{v}$ for $X(T)_{k_{v}}$.

We shall require the following basic statement from the theory of integral representations of finite groups:

Theorem 2.1. Let $G$ be a finite group and fix a positive integer d. Then there are only $O_{d}(1)$ integral representations of $G$ of dimensions d up to isomorphism.

Proof. Suppose $L$ is a $d$-dimensional integral representation of $G$. We wish to find a $G$-invariant positive-definite inner product on $L$ such that the non-zero vectors in $L$ of smallest norm generate $L$. To show this is possible, fix a $G$-invariant positivedefinite inner product $\langle,\rangle_{1}$ on $L$, and consider the span $M \subset L$ of the vectors of smallest norm. If $M$ is not all of $L$, then we can consider the dual $N$ of $M$ with respect to $\langle,\rangle_{1}$. Both $M$ and $N$ are $G$-invariant, so we can find a $G$-invariant inner product $\langle,\rangle_{2}$ which is trivial on $M$ and positive-definite on $N$. By considering $x\langle,\rangle_{1}+\langle,\rangle_{2}$ for an appropriate $x>0$, we can modify our inner product so as to strictly increase the set of non-zero vectors of smallest norm and also their span.

Thus we can find an inner product $\langle$,$\rangle on L$ which is $G$-invariant and a basis $v_{1}, \ldots, v_{n}$ consisting of elements of $L$, such that

$$
\forall i,\left\langle v_{i}, v_{i}\right\rangle=y
$$

for some $y>0$ and the norm of any other non-zero vector in $L$ is at least $y$. Let $\tilde{L}$ be the span of the $v_{i}$ (note that $\tilde{L}$ might not be $G$-invariant).

By (11) the sphere of radius $y / 2$ is contained in a fundamental domain for $L$, and the covolume of $\tilde{L}$ is at most $y^{d}$. We thus have that the index $[L: \tilde{L}]$, which is equal to the ratio of the covolumes, is at most $\frac{2^{d}}{\operatorname{Vol}\left(S^{d}\right)}$. Setting $N=\left(2^{d}\right)$ ! we see that $N \cdot L \subset \tilde{L}$. Now consider the matrix representation for an element $g \in G$ w.r.t. the basis for $v_{1}, \ldots, v_{n}$.

Set $g v_{j}=\sum_{i} a_{i j} v_{i}$. Since $N \cdot L \subset \tilde{L}$ we have $a_{i j} \in \mathbb{Z}\left[\frac{1}{N}\right]$. Since the norm of $g v$ is also $y$, by considering the basis $v_{1}, \ldots, v_{i-1}, g v, v_{i+1}, \ldots, v_{n}$ and considering covolumes we conclude as above that $\left|a_{i j}\right| \leq N$. Thus there are only $O_{d}(1)$ options for the representation of $G$ on $\tilde{L}$. Since there are only $O_{d}(1)$ options for $L$ given $\tilde{L}$, this completes the proof. 
In what follows, we write $\mathbb{A}$ for the adèle group, and $\mathbb{A}_{f}$ for the finite adèles. Likewise, for a number field $K$ we write $\mathbb{A}^{K}$ and $\mathbb{A}_{f}^{K}$ for the adèles (resp. finite adèles) over $K$.

\section{INVARIANTS OF ARITHMETIC TORI}

In this section we define several invariants of arithmetic tori and state Shyr's analogue of the class number formula. From now on we restrict to tori defined over $\mathbb{Q}$. A useful example to keep in mind is the following: Given a number field $L$, the multiplicative group $L^{\times}$can be thought of as a torus over $\mathbb{Q}$ given by restricting the multiplicative group $\mathbb{G}_{m}$ from $L$ to $\mathbb{Q}$. The corresponding character group $X(T)$ is the free $\mathbb{Z}$-module generated by the complex embeddings $\psi_{i}: L \rightarrow \mathbb{C}$ with the natural Galois action. We denote this torus by $\operatorname{Res}_{L / \mathbb{Q}} \mathbb{G}_{m}$.

3.1. Class groups and class number $h_{T}$. Define $T\left(\mathbb{Z}_{p}\right)$ to be the maximal compact subgroup of $T\left(\mathbb{Q}_{p}\right)$, and define $T(\mathbb{R})^{c}$ to be the maximal compact subgroup of $T(\mathbb{R})$. It follows that

$$
T(\hat{\mathbb{Z}}):=\prod_{p} T\left(\mathbb{Z}_{p}\right)
$$

is the maximal compact subgroup of $T\left(\mathbb{A}_{f}\right)$, and $T(\mathbb{A})^{c}:=T(\hat{\mathbb{Z}}) \times T(\mathbb{R})^{c}$ is the maximal compact subgroup of $T(\mathbb{A})$.

Define the idèle class group of $T$ to be

$$
\widetilde{C l}(T):=T(\mathbb{Q}) \backslash T\left(\mathbb{A}_{f}\right),
$$

and the regular class group of $T$ to be

$$
C l(T): T(\mathbb{Q}) \backslash T\left(\mathbb{A}_{f}\right) / T(\hat{\mathbb{Z}}) .
$$

The class number of $T$ is defined to be

$$
h_{T}:=|C l(T)| .
$$

Note that for $T=\operatorname{Res}_{L / \mathbb{Q}} \mathbb{G}_{m}$, the class number $h_{T}$ is the ordinary class number of $L$.

If $L$ is a number field, we denote by $C l(L)$ the class group of $L$. Note that if $T=\operatorname{Res}_{L / \mathbb{Q}} \mathbb{G}_{m}$, then $C l(T) \cong C l(L)$.

3.2. Units $w_{T}$ and regulator $R_{T}$. Consider a basis of $X(T)_{\infty}$ given by $\xi_{1}, \ldots, \xi_{r_{\infty}}$ such that $\xi_{1}, \ldots, \xi_{r}$ is a basis of $X(T)_{\mathbb{Q}}$. That such a basis can be chosen follows from the fact that $X(T)_{\mathbb{Q}}$ is a primitive sublattice of $X(T)$, being the fixed set of a group action. Now, the unit group $T(\mathbb{Q}) \cap T(\hat{\mathbb{Z}})$ has the decomposition

$$
T(\mathbb{Q})^{\text {tor }} \times E,
$$

where $E$ is a free abelian group of rank $r_{\infty}-r$ and $T(\mathbb{Q})^{t o r}=T(\mathbb{Q}) \cap T(\mathbb{A})^{c}$ is finite, since it is both compact and discrete in $T(\mathbb{A})$. Pick a basis $e_{i}$ for $E$. Define the number $w_{T}$ and the regulator $R_{T}$ as follows:

$$
w_{T}=\left|T(\mathbb{Q}) \cap T(\mathbb{A})^{c}\right|
$$

and

$$
R_{T}=\left|\operatorname{det}\left(\log \left|\xi_{r+i}\left(e_{j}\right)\right|\right)\right|, 1 \leq i, j \leq r_{\infty}-r .
$$

Note that for $T=\operatorname{Res}_{L / \mathbb{Q}} \mathbb{G}_{m}, w_{T}$ is the number of roots of unity in $L$, while $R_{T}$ is the regulator of $L$. 
3.3. Quasi-residue $\rho_{T}$. Let $\rho: \operatorname{Gal}(\overline{\mathbb{Q}} / \mathbb{Q}) \rightarrow G l_{d}(\mathbb{Z})$ be the representation corresponding to $T$, and let $\rho_{\mathbb{Q}}$ be the induced rational representation. We can then consider the Artin $L$-function $L\left(s, \rho_{\mathbb{Q}}\right)$. Recall that $r$ is the rank of $X(T)_{\mathbb{Q}}$ and define the quasi-residue $\rho_{T}$ of $T$ to be

$$
\rho_{T}:=\lim _{s \rightarrow 1}(s-1)^{r} L\left(s, \rho_{\mathbb{Q}}\right) .
$$

Note that for $T=\operatorname{Res}_{L / \mathbb{Q}} \mathbb{G}_{m}, \rho_{T}$ is the residue at $s=1$ of the Dedekind-Zeta function $\zeta_{L}(s)$.

3.4. Tamagawa number $\tau_{T}$. Fix an invariant gauge form $\omega$ on $T$ defined over $\mathbb{Q}$. Then $\omega$ induces canonically a Haar measure $\omega_{v}$ on $T\left(\mathbb{Q}_{v}\right)$ for each $v$. Moreover, for almost all finite primes $p$ we have $L_{p}\left(1, \rho_{\mathbb{Q}}\right) \omega_{p}\left(T\left(\mathbb{Z}_{p}\right)\right)=1$, and so

$$
\omega_{\infty} \prod_{p} L_{p}\left(1, \rho_{\mathbb{Q}}\right) \omega_{p}\left(T\left(\mathbb{Z}_{p}\right)\right)
$$

defines a Haar measure $\omega_{T}$ on $T(\mathbb{A})$, which is moreover independent of the original choice of $\omega$ by the product formula.

Define $T(\mathbb{A})^{1}$ to be the kernel of the map $\Lambda: T(\mathbb{A}) \rightarrow\left(\mathbb{R}_{+}^{\times}\right)^{r}$ given by

$$
\Lambda(x)=\left(\left\|\xi_{i}(x)\right\|_{\mathbb{Q}}\right)_{1 \leq i \leq r},
$$

where $\|\cdot\|_{\mathbb{Q}}$ denotes the idèle volume of $\mathbb{Q}$. Pulling back the measure $\Lambda_{i=1}^{r} t_{i}^{-1} d t_{i}$ gives us a measure $d \tilde{t}$ on $T(\mathbb{A}) / T(\mathbb{A})^{1}$. Let $m$ be the measure on $T(\mathbb{A})^{1}$ such that $m$ and $d \tilde{t}$ glue together to give $\rho_{T}^{-1} \omega_{T}$. We define the Tamagawa number $\tau_{T}$ to be the measure of $T(\mathbb{A})^{1} / T(\mathbb{Q})$ under $m$.

For $T=\operatorname{Res}_{L / \mathbb{Q}} \mathbb{G}_{m}$, the Tamagawa number is 1 .

3.5. Quasi-discriminant $D_{T}$. Assigning $T(\mathbb{R})^{c}$ measure 1 and gluing it with the measure $\Lambda_{i=1}^{r_{\infty}} d \xi_{i}$ on $T(\mathbb{R}) / T(\mathbb{R})^{c}$ we get a measure on $T(\mathbb{R})$, which we also denote by $d \tilde{t}$. Now, define

$$
c_{T}=\frac{w_{\infty}}{d \tilde{t}} \prod_{p} L_{p}\left(1, \rho_{\mathbb{Q}}\right) \omega_{p}\left(T\left(\mathbb{Z}_{p}\right)\right) .
$$

The quasi-discriminant $D_{T}$ is defined to be $D_{T}=1 / c_{T}^{2}$.

For $T=\operatorname{Res}_{L / \mathbb{Q}} \mathbb{G}_{m}$ the quasi-discriminant $D_{T}$ is the ordinary discriminant $D_{L}$.

3.6. Analytic class number formula for tori. We have the following result of Shyr [14]:

Theorem 3.1 (Shyr). The following equality holds:

$$
h_{T} \cdot R_{T}=\rho_{T} \cdot w_{T} \cdot \tau_{T} \cdot\left|D_{T}\right|^{1 / 2} .
$$

For $T=\operatorname{Res}_{L / \mathbb{Q}} \mathbb{G}_{m}$, this reduces to Dirichlet's class number formula for $L$.

\section{BRAUER-SiEgEl FORMUla FOR TORI}

Define $K$ to be the splitting field of $T$, and let $D_{K}$ be its discriminant. Set $d$ to be the dimension of $T$ and $n$ to be $[K: \mathbb{Q}]$. In this section we use Shyr's class number formula to derive a Brauer-Siegel theorem for tori by estimating the sizes of the invariants $w_{T}, \rho_{T}, \tau_{T}$ and $D_{T}$ with respect to $D_{K}$. We show that except for $D_{T}$, the invariants are all small. To make this precise, we find it useful also to 
introduce the following notation: A function $F(T, K)$ is said to be discriminant negligible if $\forall \epsilon>0$, we have

$$
D_{K}^{-\epsilon} \ll_{n, d, \epsilon}|F(K, T)| \ll_{n, d, \epsilon} D_{K}^{\epsilon} .
$$

Lemma 4.1. $\rho_{T}$ is discriminant negligible.

Remark. This is the only part of the paper which is ineffective. That is, we cannot write down a $C_{\epsilon, n, d}>0$ such that

$$
\frac{1}{C_{\epsilon, n, d}} \cdot D_{K}^{-\epsilon} \leq|F(K, T)| \leq C_{\epsilon, n, d} \cdot D_{K}^{\epsilon} \cdot
$$

This stems from Siegel's ineffective lower bound for $L(1, \chi)$, where $\chi$ is a quadratic Dirichlet character, and is the reason why the Brauer-Siegel theorem, Theorem 4.7. and our lower bounds for Galois orbits of CM points are all ineffective.

Proof. Let $L$ be a subfield of $K$ such that $\operatorname{Gal}(K / L)$ is Galois and let $\chi$ be a character of $\operatorname{Gal}(L / \mathbb{Q})$. Then the estimate

$$
D_{K}^{-\epsilon} \ll_{\epsilon} L(\chi, 1) \ll_{\epsilon} D_{K}^{\epsilon}
$$

is known by Siegel's work. By Brauer's induction theorem, $L\left(\rho_{\mathbb{Q}}, 1\right)$ can be expressed as a quotient of products of such $L$-functions, and the result follows.

Lemma 4.2. $\tau_{T}=O_{n, d}(1)$, and $1 / \tau_{T}=O_{n, d}(1)$. Therefore $\tau_{T}$ is discriminant negligible.

Proof. The Tate-Shafarevich group $\amalg_{T}$ is defined to be the kernel of the map

$$
H^{1}(\operatorname{Gal}(\overline{\mathbb{Q}} / \mathbb{Q}), T(\mathbb{Q})) \rightarrow \prod_{p} H^{1}\left(\operatorname{Gal}\left(\overline{\mathbb{Q}_{p}} / \mathbb{Q}_{p}, T\left(\mathbb{Q}_{p}\right)\right)\right.
$$

A result of Ono [9] says that

$$
\tau_{T}=\frac{\left|\amalg_{T}\right|}{\left|H^{1}(\operatorname{Gal}(K / \mathbb{Q}), X(T))\right|} .
$$

Now, since we are fixing $d$ and $n$, the group $\operatorname{Gal}(K / \mathbb{Q})$ is in one of a finite number of isomorphism classes of groups and by Theorem 2.1 the pair $(\operatorname{Gal}(K, \mathbb{Q}), X(T))$ goes over a finite set of isomorphism types of $(G, M)$, where $G$ is a group and $M$ is a representation of $G$.

Therefore $\left|H^{1}(\operatorname{Gal}(K / \mathbb{Q}), X(T))\right|$ is $O_{n, d}(1)$. Likewise, as stated in the proof of ([12], Prop. 6.9), the group $\amalg_{T}$ is a quotient of $H^{2}(\operatorname{Gal}(K / \mathbb{Q}), X(T))$ and so its order is $O_{n, d}(1)$ by the same argument.

Lemma 4.3. The order of the group of units satisfies $w_{T}=O_{n, d}(1)$, and so $w_{T}$ is discriminant negligible.

Note that $T(\mathbb{Q})^{\text {tor }}$ is a subgroup of $T(K)^{\text {tor }} \approx\left(\left(K^{\times}\right)^{\text {tor }}\right)^{n}$. The degree of a primitive $q$ th root of unity over $\mathbb{Q}$ is $\phi(q)$. Since $\phi(q)$ goes to infinity with $q$ and the degree of $K$ is $d$, the order of the unit group of $K$ is $O_{d}(1)$. The result follows.

Lemma 4.4. Let $T$ and $T^{\prime}$ be isogenous tori in $C(K / \mathbb{Q})$ of dimension $d$. Then $D_{T}=D_{T^{\prime}} \cdot O_{\epsilon, d, n}\left(D_{K}^{\epsilon}\right)$. 
Proof. By Lemma[2.1 there exists an isogeny $\lambda: T \rightarrow T^{\prime}$ between $T$ and $T^{\prime}$ of degree $m$, where $m=O_{d, n}(1)$. For each finite prime $p$ this induces a map $\lambda_{p}^{c}: T\left(\mathbb{Z}_{p}\right) \rightarrow$ $T^{\prime}\left(\mathbb{Z}_{p}\right)$. Define $q\left(\lambda_{p}^{c}\right)=\frac{\left|\operatorname{Coker}\left(\lambda_{p}^{c}\right)\right|}{\left|\operatorname{Ker}\left(\lambda_{p}^{c}\right)\right|}$. Then by the discussion at the beginning of section 4 of [14], we have

$$
D_{T} / D_{T^{\prime}}=\prod_{p} q\left(\lambda_{p}^{c}\right)
$$

Now, as Shyr points out at the end of section 2 of [14], $q\left(\lambda_{p}^{c}\right)=1$ for all primes $p$ that are unramified in $K$ and satisfy $(p, m)=1$. Since $\lambda$ is an isogeny of degree $m$, it factors through the $\times m$ map, and so we have

$$
\left|\operatorname{Coker}\left(\lambda_{p}^{c}\right)\right| \leq\left|T^{\prime}\left(\mathbb{Z}_{p}\right)[m]\right| \leq\left|T^{\prime}\left(\overline{\mathbb{Q}_{p}}\right)[m]\right|=m^{d} .
$$

Setting $S$ to be the set of all primes ramified in $K$, we thus have

$$
D_{T}=D_{T^{\prime}} \times O_{d, n}\left(m^{d|S|}\right),
$$

from which the result follows.

Corollary 4.5. Let $T$ be a torus in $C(K / \mathbb{Q})$ of dimension d. Let $\rho$ be the associated integral Galois representation, and $\rho_{\mathbb{Q}}$ the induced rational representation. Let $f_{\rho}$ be the norm of the Artin conductor corresponding to $\rho_{\mathbb{Q}}$. Then $\frac{f_{\rho}}{D_{T}}$ is discriminant negligible.

Proof. For tori of the form $R_{L / \mathbb{Q}}\left(\mathbb{G}_{m}\right)$, where $L$ is a subfield of $K$, we actually have equality since the Artin conductor and the quasi-discriminant are both equal to the discriminant of $L$. By Theorem 1.5.1 in [10] there are integers $m, m_{i}, n_{i}$ of size $O_{n, d}(1)$ such that $T^{m} \times \prod_{i}\left(R_{L_{i} / \mathbb{Q}}\left(\mathbb{G}_{m}\right)\right)^{m_{i}}$ is isogenous to $\prod_{i}\left(R_{L_{i} / \mathbb{Q}}\left(\mathbb{G}_{m}\right)\right)^{n_{i}}$ where $L_{i}$ goes over all subfields of $K$. Since both the quasi-discriminant and the Artin conductor are multiplicative under products, the corollary now follows from Lemma 4.4.

The following lemma is essential in showing that the class number of tori is large in terms of the discriminant of the splitting field.

Lemma 4.6. If $K$ is a Galois extension of $\mathbb{Q}$ of degree $n$ and $\rho: \operatorname{Gal}(K / Q) \rightarrow$ $G L(V)$ is a representation of $\operatorname{Gal}(K / \mathbb{Q})$ on a complex vector space $V$ with trivial kernel, then

$$
f_{\rho} \gg_{n} D_{K}^{\frac{1}{n-1}}
$$

Proof. Let $\rho: \operatorname{Gal}(K / \mathbb{Q}) \rightarrow G L(V)$. Since $\rho$ has trivial kernel, it is non-trivial on all non-trivial inertia groups. Let $I_{p}$ denote the inertia group of a prime over $p$. Since $v_{p}\left(f_{\rho}\right) \geq \operatorname{dim}_{\mathbb{C}}\left(V / V^{I_{p}}\right)$, each ramified prime $p$ of $K$ divides $f_{\rho}$. Now, each prime which is tamely ramified divides $D_{K}$ with exponent at most $n-1$. Since the contribution of the wildly ramified primes is a priori bounded by the dimension, we have

$$
D_{K} \ll_{n} \prod_{p} p^{n-1},
$$

where the product is over ramified primes in $K$. This implies the lemma. 
Putting together all the above we arrive at

Theorem 4.7 (Brauer-Siegel Theorem for tori). Let $T$ be a torus of dimensiond in $C(K / \mathbb{Q})$ with $[K: \mathbb{Q}]=n$, with $K$ being the minimal splitting field of $T$. Let $h_{T}, R_{T}$ be the class number and regulator of $T$ respectively. Also, let $\rho$ be the associated integral representation of $\mathrm{Gal}(\overline{\mathbb{Q}} / \mathbb{Q})$ and $\rho_{\mathbb{Q}}$ the induced rational representation. Let $f_{\rho}$ be the norm of the Artin conductor corresponding to $\rho_{\mathbb{Q}}$. Then we have

$$
\begin{aligned}
\frac{1}{2} \ln \left(h_{T} R_{T}\right) & =\frac{1}{2} \ln f_{\rho}+o_{n, d}\left(\ln D_{K}\right) \\
& =\left(\frac{1}{2}+o_{n, d}(1)\right) \ln f_{\rho} .
\end{aligned}
$$

Proof. The first equality is an immediate consequence of the previous lemmas and Theorem 3.1. The second equality follows from Lemma 4.6 and the fact that $K$ is the minimal splitting field for $T$.

\section{Structure theOREMS FOR Class GROUPS OF TORI}

We focus in this section on the following setup: Suppose we have a map $\phi: S \rightarrow T$ of tori defined over $\mathbb{Q}$. This then induces a map of class groups $\phi_{0}: C l(S) \rightarrow C l(T)$, and we wish to understand how large the image of $\phi_{0}$ is. This question can be very difficult. For instance, suppose that $S \cong T$ and $\phi$ is the map sending $t$ to $t^{n}$. Then the question reduces to understanding the size of the group $C l(T)[n]$, and bounding torsion for class groups of number fields is notoriously difficult. We show, however, that this is essentially the only hard case. More precisely, we prove the following theorem:

Theorem 5.1. Let

$$
1 \longrightarrow S \stackrel{f}{\longrightarrow} T \stackrel{g}{\longrightarrow} U \longrightarrow 1
$$

be an exact sequence of tori, where $S, T, U$ are in $C(K / \mathbb{Q}),[K: \mathbb{Q}]=n$ and the dimension of $T$ is $d$. Then the homology groups of the induced sequence

$$
1 \longrightarrow \mathrm{Cl}(S) \stackrel{f_{0}}{\longrightarrow} \mathrm{Cl}(\mathrm{T}) \stackrel{g_{0}}{\longrightarrow} \mathrm{Cl}(U) \longrightarrow 1
$$

are discriminant negligible.

Proof. For a torus $T$, recall that $\widetilde{C l}(T)$ denotes $T(\mathbb{Q}) \backslash T\left(\mathbb{A}_{f}\right)$. By considering the cohomology sequence associated to the exact sequence

$$
1 \longrightarrow T(K) \longrightarrow T\left(\mathbb{A}^{K}\right) \longrightarrow T(K) \backslash T\left(\mathbb{A}^{K}\right) \longrightarrow 1
$$

we see that there is an injection

$$
\psi_{T}: \widetilde{C l}(T) \hookrightarrow H^{0}\left(\operatorname{Gal}(K / \mathbb{Q}), T(K) \backslash T\left(\mathbb{A}^{K}\right)\right) .
$$

Moreover, the image contains the image of the norm map on $T(K) \backslash T\left(\mathbb{A}^{K}\right)$ and thus the size of the cokernel of $\psi_{T}$ is at most the size of the Tate cohomology group $\hat{H}^{0}\left(\operatorname{Gal}(K / \mathbb{Q}), T(K) \backslash T\left(\mathbb{A}^{K}\right)\right)$. By Nakayama-Tate duality this is isomorphic to $H^{2}(\operatorname{Gal}(K / \mathbb{Q}), X(T))$ which is $O_{n, d}(1)$ by Lemma 2.1 as has already been discussed.

Now, suppose we have an exact sequence of tori

$$
1 \longrightarrow S \stackrel{f}{\longrightarrow} T \stackrel{g}{\longrightarrow} U \longrightarrow 1
$$


as in the statement. We first prove that $g_{0}$ has a small cokernel, and then that $f_{0}$ has a small kernel. The theorem then follows since $h_{T}\left(h_{S} h_{U}\right)^{-1}$ is discriminant negligible by Lemma 4.7

- $g_{0}$ has small cokernel:

Since all our tori become split over $K$, we have an exact sequence

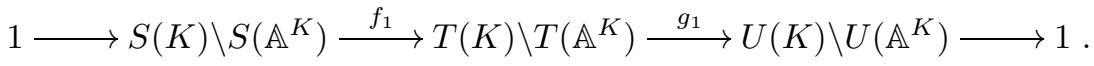

Taking cohomology, we get an exact sequence

$$
H^{0}\left(G, T(K) \backslash T\left(\mathbb{A}^{K}\right)\right) \longrightarrow H^{0}\left(G, U(K) \backslash U\left(\mathbb{A}^{K}\right)\right) \longrightarrow H^{1}\left(G, S(K) \backslash S\left(\mathbb{A}^{K}\right)\right),
$$

where we set $G=\operatorname{Gal}(K / \mathbb{Q})$. By Nakayama-Tate duality,

$$
H^{1}\left(\operatorname{Gal}(K / \mathbb{Q}), S(K) \backslash S\left(\mathbb{A}^{K}\right)\right) \cong H^{1}(\operatorname{Gal}(K / \mathbb{Q}), X(S))
$$

and is thus of size $O_{n, d}(1)$. Consider the commutative diagram

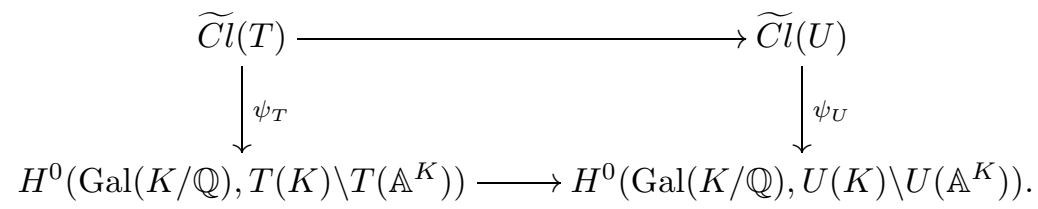

The downward maps are injective with cokernels of size $O_{n, d}(1)$ and the bottom map has cokernel of size $O_{n, d}(1)$, so the map $\widetilde{C l}(T) \rightarrow \widetilde{C l}(U)$ must also have cokernel of size $O_{n, d}(1)$. Finally, consider the commutative diagram

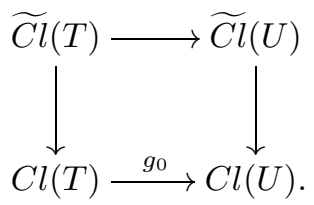

The top map has cokernel of size $O_{n, d}(1)$ and the downwards maps are surjective, so we see that $g_{0}$ also has cokernel of size $O_{n, d}(1)$.

- $f_{0}$ has small kernel:

For a ring $R$, we consider $S(R)$ as a subgroup of $T(R)$. We have

$$
\operatorname{ker}\left(f_{0}\right) \approx \frac{T(\hat{\mathbb{Z}}) T(\mathbb{Q}) \cap S\left(\mathbb{A}_{f}\right)}{S(\hat{\mathbb{Z}}) S(\mathbb{Q})} .
$$

By group theory we have:

$$
\left|\frac{T(\hat{\mathbb{Z}}) T(\mathbb{Q}) \cap S\left(\mathbb{A}_{f}\right)}{S(\hat{\mathbb{Z}}) S(\mathbb{Q})}\right| \leq\left|\frac{T(\hat{\mathbb{Z}}) \cap S\left(\mathbb{A}_{f}\right)}{S(\hat{\mathbb{Z}})}\right| \cdot\left|\frac{T(\hat{\mathbb{Z}}) S\left(\mathbb{A}_{f}\right) \cap T(\mathbb{Q})}{S(\mathbb{Q})(T(\hat{\mathbb{Z}}) \cap T(\mathbb{Q}))}\right| .
$$

The first term on the right-hand side is 1 since $S(\hat{\mathbb{Z}})$ is the maximal compact subgroup of $S\left(\mathbb{A}_{f}\right)$. For the second part, consider the map $g$ from $T\left(\mathbb{A}_{f}\right)$ to $U\left(\mathbb{A}_{f}\right)$. Since the kernel of $g$ is $S\left(\mathbb{A}_{f}\right)$ and

$$
T(\mathbb{Q}) \cap S\left(\mathbb{A}_{f}\right)=S(\mathbb{Q}),
$$


this induces an injective map $\tilde{g}$ on

$$
\frac{T(\hat{\mathbb{Z}}) S\left(\mathbb{A}_{f}\right) \cap T(\mathbb{Q})}{S(\mathbb{Q})(T(\hat{\mathbb{Z}}) \cap T(\mathbb{Q}))} .
$$

The image of $g$ on $T(\hat{\mathbb{Z}}) S\left(\mathbb{A}_{f}\right) \cap T(\mathbb{Q})$ lies inside $U(\mathbb{Q}) \cap U(\hat{\mathbb{Z}})$. Now,

$$
U(\mathbb{Q}) \cap U(\hat{\mathbb{Z}})=U(\mathbb{Q})^{\text {tor }} \times E_{U},
$$

where $E_{U}$ is a finitely generated free abelian group of rank at most $d$. Also, corresponding to the map $g: T \rightarrow U$ there is a map $h: U \rightarrow T$ such that $g \circ h$ is the multiplication by $m$ map on $U$, where $m=O_{n, d}(1)$. This implies that the image of $g$ on $S(\mathbb{Q})(T(\hat{\mathbb{Z}}) \cap T(\mathbb{Q}))$ is at least the image of the multiplication by $m$ map on $E_{U}$. Therefore, the size of the image of $\tilde{g}$ is bounded by $\left|E_{U}[m]\right| \cdot\left|U(\mathbb{Q})^{t o r}\right| \leq m^{d} \cdot w_{U}$, which means $\operatorname{ker}\left(f_{0}\right)=O_{d}(1)$, as desired.

As a by-product of the above, we get the following important corollary, which can be considered as a very general transfer principle for torsion in class groups.

Corollary 5.2. Let $M$ be a fixed finite abelian group on which $\operatorname{Gal}(K / \mathbb{Q})$ acts, where $[K: \mathbb{Q}]$ is bounded by $n$. Let $T_{1}, T_{2}, S_{1}, S_{2}$ be tori which split over $K$ whose dimensions are bounded by $d$. Let $\phi_{i}: T_{i} \rightarrow S_{i}, i=1,2$ be maps which are surjective as maps of tori, and such that the cokernel of $X\left(T_{i}\right)^{*} \rightarrow X\left(S_{i}\right)^{*}$ is isomorphic to $M$ as a $\operatorname{Gal}(K / \mathbb{Q})$ module. Finally, let $C_{i}$ be the size of the cokernel of the induced map $\widetilde{\phi}_{i}: C l\left(T_{i}\right) \rightarrow C l\left(S_{i}\right)$. Then $\frac{C_{1}}{C_{2}}$ is discriminant negligible.

Proof. We shall work with cocharacters for the purpose of this proof. We have maps $\psi_{i}: X\left(S_{i}\right)^{*} \rightarrow M$ whose kernels are $\phi_{i}\left(X\left(T_{i}\right)^{*}\right)$. Let

$$
L_{S} \subset X\left(S_{1}\right)^{*} \oplus X\left(S_{2}\right)^{*}
$$

be the kernel of the map

$$
\Phi: X\left(S_{1}\right)^{*} \oplus X\left(S_{2}\right)^{*} \rightarrow M
$$

defined by $\Phi(a, b)=\psi_{1}(a)+\psi_{2}(b)$, and let $U$ be the torus whose cocharacter module is $L_{S}$. We then have the following commutative diagram:

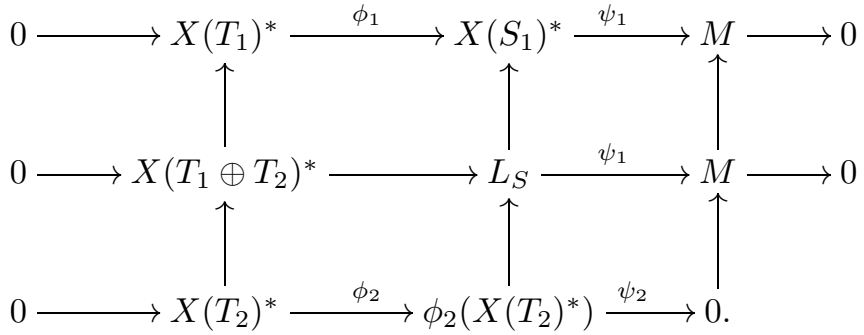

The vertical and horizontal sequences are exact. Now consider the induced diagram on class groups. Since $\phi_{2}$ is surjective, it follows from Theorem 5.1 that the induced map on class groups is surjective up to discriminant negligible factors. Also, the induced vertical sequences are exact up to discriminant negligible factors. Let $C$ be the size of the cokernel of $C l\left(T_{1}\right) \oplus C l\left(T_{2}\right) \rightarrow C l(U)$. An application of the snake lemma shows that $\frac{C_{1}}{C}$ is discriminant negligible. Likewise, $\frac{C_{2}}{C}$ is discriminant negligible. The result follows. 
We denote the size of the above cokernels by $|C k(M)|$. This quantity is well defined only up to a discriminant negligible factor. We formulate below a simple lemma that we will use in the subsequent sections:

Lemma 5.3. Let $0 \rightarrow M_{1} \rightarrow M \rightarrow M_{2} \rightarrow 0$ be an exact sequence of finite $\operatorname{Gal}(K / \mathbb{Q})$ modules. Then

$$
\max \left(\left|C k\left(M_{1}\right)\right|,\left|C k\left(M_{2}\right)\right|\right) \leq|C k(M)| \leq\left|C k\left(M_{1}\right)\right| \cdot\left|C k\left(M_{2}\right)\right| .
$$

Proof. Consider $\operatorname{Gal}(K / \mathbb{Q})$-lattices $L_{1}, L_{2}$ such that $L_{1} / L_{2} \approx M$. By pulling back $M_{1}$ we can find a lattice $L_{3}$ containing $L_{2}$ and contained in $L_{1}$ such that $L_{3} / L_{2} \approx$ $M_{1}$ and $L_{1} / L_{2} \approx M_{2}$. Let $S, T, U$ be the tori whose cocharacter modules are $L_{1}, L_{2}, L_{3}$ respectively. We have maps $f: T \rightarrow U$ and $g: U \rightarrow S$ corresponding to the inclusion of the cocharacter modules. This induces maps on class groups:

$$
C l(T) \stackrel{f}{\longrightarrow} C l(U) \stackrel{g}{\longrightarrow} C l(S) .
$$

By Corollary 5.2 we have $|\operatorname{ker}(f \circ g)| \approx|C k(M)|,|\operatorname{ker}(f)| \approx\left|C k\left(M_{1}\right)\right|$, and $|\operatorname{ker}(g)|$ $\approx\left|C k\left(M_{2}\right)\right|$ up to discriminant negligible factors. Moreover, since the tori are all isogenous their class groups have the same size up to discriminant negligible factors by Theorem 4.7. The result follows.

As an important special case, suppose $M$ is a finite Galois module with a trivial Galois action. Then $M$ can be expressed as a quotient of $\mathbb{Z}^{n}$ for some integer $n$, where we consider $\mathbb{Z}^{n}$ as a Galois module with a trivial Galois action. $\mathbb{Z}^{n}$ is the cocharacter module of $\left(\mathbb{G}_{m}\right)^{n}$, and since the class number of $\mathbb{Q}$ is 1 , we conclude the following.

Corollary 5.4. Let $M$ be a finite module with a trivial action of the Galois group. Then $|C k(M)|$ is discriminant negligible.

We now give a further interpertation of the class groups of tori, and of the quantities $|C k(M)|$. Though not necessary for the main results of this paper, we feel that the next theorem and its corollary shed light on the nature of these objects.

Suppose $T$ is a torus of dimension $d$ in $C(L / \mathbb{Q}),[L: \mathbb{Q}]=n$. Denote the Galois group of $L$ over $\mathbb{Q}$ by $G$. We then have natural isomorphisms:

$$
\begin{aligned}
T(\mathbb{Q}) & \cong \operatorname{Hom}_{G}(X(T), T(L)), \\
T\left(\mathbb{A}_{f}\right) & \cong \operatorname{Hom}_{G}\left(X(T), T\left(\mathbb{A}_{f}^{L}\right)\right), \\
T(\widehat{\mathbb{Z}}) & \cong \operatorname{Hom}_{G}\left(X(T), T\left(\widehat{\mathcal{O}}_{L}\right)\right) .
\end{aligned}
$$

We thus have a natural map from $C l(T)$ to $\operatorname{Hom}_{G}(X(T), C l(L))$.

Theorem 5.5. Let $T$ be a torus of dimension $d$ in $C(L / \mathbb{Q})$. Denote the Galois group of $L$ over $\mathbb{Q}$ by $G$. The natural map from $C l(T)$ to $\operatorname{Hom}_{G}(X(T), C l(L))$ has discriminant negligible kernel and cokernel.

Proof. Let $U_{L}$ denote the unit group of $L$. We have the following exact sequence:

$$
1 \rightarrow \operatorname{Hom}\left(X(T), \widehat{\mathcal{O}}_{L} / U_{L}\right) \rightarrow \operatorname{Hom}\left(X(T), \mathbb{A}_{f}^{L} / L^{\times}\right) \rightarrow \operatorname{Hom}(X(T), C l(L)) \rightarrow 1,
$$

or equivalently

$$
1 \rightarrow \operatorname{Hom}\left(X(T), \widehat{\mathcal{O}}_{L} / U_{L}\right) \rightarrow T\left(\mathbb{A}_{f}^{L}\right) / T(L) \rightarrow \operatorname{Hom}(X(T), C l(L)) \rightarrow 1 .
$$


Taking $G$-invariants gives the associated exact sequence

$$
\begin{aligned}
& 1 \longrightarrow \operatorname{Hom}_{G}\left(X(T), \widehat{\mathcal{O}}_{L} / U_{L}\right) \longrightarrow\left(T\left(\mathbb{A}_{f}^{L}\right) / T(L)\right)^{G} \longrightarrow H^{1}\left(\widehat{\mathcal{O}}_{L} / U_{L}\right) \longrightarrow 1 \\
& \longrightarrow \operatorname{Hom}_{G}(X(T), C l(L)) \longrightarrow
\end{aligned}
$$

Now, there is an embedding of $T\left(\mathbb{A}_{f}\right) / T(\mathbb{Q})$ into $\left(T\left(\mathbb{A}_{f}^{L}\right) / T(L)\right)^{G}$ and NakayamaTate duality implies that the cokernel is discriminant negligible, as in shown in the proof of Theorem 5.1. We now analyze the second term $\widehat{\mathcal{O}}_{L} / U_{L}$. Consider the exact sequence

$$
1 \rightarrow \operatorname{Hom}\left(X(T), U_{L}\right) \rightarrow T\left(\mathbb{A}_{f}^{L}\right)^{c} \rightarrow \operatorname{Hom}\left(X(T), \widehat{\mathcal{O}}_{L} / U_{L}\right) \rightarrow 1 .
$$

Taking $G$-invariants gives us the exact sequence

$$
\begin{aligned}
1 & \rightarrow T(\mathbb{Q}) \cap T(\hat{\mathbb{Z}}) \rightarrow T(\hat{\mathbb{Z}}) \rightarrow \operatorname{Hom}_{G}\left(X(T), \widehat{\mathcal{O}}_{L} / U_{L}\right) \\
& \rightarrow H^{1}\left(\operatorname{Hom}\left(X(T), U_{L}\right)\right) \rightarrow H^{1}\left(T\left(\mathbb{A}_{f}^{L}\right)^{c}\right) \rightarrow H^{1}\left(\operatorname{Hom}\left(X(T), \widehat{\mathcal{O}}_{L} / U_{L}\right)\right) \\
& \rightarrow H^{2}(\operatorname{Hom}(X(T), U(L))) .
\end{aligned}
$$

As $U_{L}$ is a finitely generated abelian group of rank bounded by the degree of $L$, all its cohomology groups are of bounded cardinality. The theorem thus follows from the following lemma:

Lemma 5.6. The cohomology group $H^{1}\left(T\left(\widehat{\mathcal{O}}_{L}\right)\right)$ is finite and discriminant negligible.

Proof. Note that we have the decomposition

$$
H^{1}\left(T\left(\widehat{\mathcal{O}}_{L}\right)\right) \cong \bigoplus_{v} H^{1}\left(T\left(\mathcal{O}_{L, v}\right)\right)
$$

Let $v$ be a place lying over a finite prime $p$ such that it is unramified. Then by local class field theory, $\mathcal{O}_{L, v}$ is a cohomologically trivial module, and thus by Nakayama ([8], Th. 2) $H^{1}\left(T\left(\mathcal{O}_{L, v}\right)\right)$ is trivial.

If $v$ is ramified, consider the exact sequence

$$
1 \rightarrow T\left(\mathcal{O}_{L, v}\right) \rightarrow T\left(L_{v}\right) \rightarrow X(T)^{*} \rightarrow 1 .
$$

By Nakayama-Tate duality, the cohomology groups of $T\left(L_{v}\right)$ are bounded in terms of the degree of $L$. By looking at the associated long exact sequence, it thus follows that the same holds for $T\left(\mathcal{O}_{L_{v}}\right)$. Let $S$ be the set of primes ramified in $L$. The lemma now follows from the fact that $2^{|S|}$ is discriminant negligible.

We have the following corollary:

Corollary 5.7. Let $M$ be a finite module of the Galois group $\operatorname{Gal}(\overline{\mathbb{Q}} / \mathbb{Q})$, which factors through the Galois group $G$ of a number field $L$. Let $M^{*}$ denote the dual $\operatorname{module} \operatorname{Hom}(M, \mathbb{Q} / \mathbb{Z})$. Then up to discriminant negligible factors, we have the equality

$$
|C l(M)|=\left|\operatorname{Hom}_{G}\left(M^{*}, C l(L)\right)\right| .
$$

Proof. Let $\phi: T \rightarrow S$ be a map of isogenous tori that split over $L$ such that the cokernel of the induced map $X\left(T^{*}\right) \rightarrow X\left(S^{*}\right)$ is $M$. This implies that the cokernel of the map $X(S) \rightarrow X(T)$ is $M^{*}$. 
By Theorem [5.5] up to discriminant negligible factors, $C l(M)$ is the size of the kernel of the map $\operatorname{Hom}_{G}(X(T), C l(L)) \rightarrow \operatorname{Hom}_{G}(X(S), C l(L))$, which is exactly $\operatorname{Hom}_{G}\left(M^{*}, C l(L)\right)$.

\section{General transfer PRINCiPles FOR Class Groups}

We can apply Corollary 5.2 to derive transfer principles for torsion in class groups of number fields in the following way: Take $K$ to be an algebraic number field and consider the multiplication by $n$ map on $T=\operatorname{Res}_{K / \mathbb{Q}} \mathbb{G}_{m}$. The cokernel of the induced map on class groups is precisely $C l(K)[n]$, so if we let $M$ be the finite module $X(T)^{*} / n X(T)^{*}$, then $|C k(M)|=|C l(K)[n]|$.

As an example, consider a cubic field $K$ that is not normal. Let $L$ be its Galois closure, and $k$ the quadratic subfield of $L$. This is called the quadratic resolvent of $K$. Then a result of Gerth [6] says that the 3-torsion in $C l(K)$ and $C l(k)$ are the same up to a factor of $O_{\epsilon}\left(D_{K}^{\epsilon}\right)$. We can recover this result as follows:

Identify the Galois group $G=\operatorname{Gal}(L / \mathbb{Q})$ with $S_{3}$. The 3-torsion in $C l(K)$ corresponds to the $G$-module $M$ generated by $a_{1}, a_{2}, a_{3}$ over $\mathbb{F}_{3}$ with the natural permutation action by $S_{3}$, while the 3 -torsion in $C l(k)$ corresponds to a $G$-module $N$, which is isomorphic to the submodule of $M$ generated by $a_{1}-a_{2}$ and $a_{2}-a_{3}$. Moreover, $M / N$ is a trivial $G$-module. By Corollary [5.4 we have that $|C k(M / N)|$ is discriminant negligible, and so the result follows by Lemma 5.3

Similarly, suppose $K$ is a quartic field whose normal closure $\tilde{K}$ has $S_{4}$ Galois group over $\mathbb{Q}$. There are 3 subgroups of $S_{4}$ of order 8 , all conjugate and isomorphic to the dihedral group with 8 elements $D_{4}$. Pick one of these, and let $F$ be its fixed field. $F$ is called the cubic resolvent field of $K$. We have the following result relating 2 -torsion in quartic fields to 2-torsion in the corresponding cubic resolvents. This result is new as far as the author knows:

Lemma 6.1. Let $K$ be a quartic field, and $F$ be its cubic resolvent. Then the 2-torsion in $C l(K)$ and in $C l(F)$ are the same up to a factor of size $O_{\epsilon}\left(D_{K}^{\epsilon}\right)$.

Proof. Let $L$ be the Galois closure of $K$ and assume for simplicity that $G=$ $\operatorname{Gal}(L / \mathbb{Q}) \approx S_{4}$. Let $M$ and $N$ be the $G$-modules corresponding to $C l(K)[2]$ and $C l(F)[2]$ respectively. As before, $M$ corresponds to the $\mathbb{F}_{2}$ vector space generated by $a_{1}, a_{2}, a_{3}, a_{4}$ with the natural permutation action of $S_{4}$. We have a 1 -dimensional trivial submodule $M_{0}$ of $M$ generated by $a_{1}+a_{2}+a_{3}+a_{4}$. Set $M_{1}$ to be $M / M_{0}$. Now, let $M_{2}$ be the submodule of $M_{1}$ generated by $a_{1}+a_{2}, a_{1}+a_{3}$, so that $M_{1} / M_{2}$ is a trivial $G$-module. A basis for $N$ as a module over $\mathbb{F}_{2}$ is given by $b_{1}, b_{2}, b_{3}$, where the $S_{4}$ action is given by identifying the $b_{i}$ with the right cosets $S_{4} / D_{4}$. We have a trivial 1-dimensional submodule $N_{0}$ given by $b_{1}+b_{2}+b_{3}$, and $N / N_{0} \approx M_{2}$ as $S_{4}$-modules. Now, by applying Corollaries 5.3 and 5.4 we get, up to discriminant negligible factors, the equalities

$$
|C k(M)|=\left|C k\left(M_{1}\right)\right|=\left|C k\left(M_{2}\right)\right|=\left|C k\left(N / N_{0}\right)\right|=|C k(N)|,
$$

as desired.

We end this section with some general remarks on torsion in class groups. One expects that all torsion is discriminant negligible. For positive integers $n, d$, Zhang [18] conjectures the bound

$$
|C l(K)[n]| \ll_{\epsilon} D_{K}^{\epsilon}
$$


as $K$ varies over number fields of degree $d$. We can phrase Zhang's conjecture in our language in the following way: Fix a group $G$ and a finite $G$-module $M$. Now, suppose $K$ is a Galois number field with $\operatorname{Gal}(K / \mathbb{Q}) \approx G$. We can then consider $M$ as a finite $\operatorname{Gal}(K / \mathbb{Q})$ module. It then makes sense to talk about $|C k(M)|$, leaving $K$ out by abuse of notation.

Conjecture 6.1. For all finite modules $M$, the function $|C k(M)|$ is discriminant negligible.

It is not hard to see that Conjecture 6.1 is equivalent to Zhang's conjecture. Zhang's conjecture is the special case where the module $M$ is the regular representation of $G$ over $\mathbb{Z} / n \mathbb{Z}$ for some positive integer $n$. However, since all modules are quotients of direct sums of such regular representations, the equivalence follows from Corollary 5.3

- In light of Conjecture 6.1, the result of Gerth and Lemma 6.1 might not seem that interesting, since all torsion is supposed to be discriminant negligible! In fact, Gerth [6] proves a much more precise relationship between the 3-torsion group of a cubic field and its quadratic resolvent, in terms of ramification. By following the proof of Theorem 5.1 more closely, one can prove that $|C k(M)|$ is well defined up to, roughly speaking, the contribution from the ramified primes in $K$. Thus, the statement of Lemma 6.1 could likely be refined to say that the 2-ranks of a quartic field and its cubic resolvent are the same up to ramified primes. As our main interest is in Galois orbits of CM points, we do not pursue this.

- In fact, Brumer and Silverman [1] asked whether the right-hand side of (3) can be replaced with $D_{K}^{\frac{C}{\ln \ln D_{K}}}$. The value $D_{K}^{\frac{C}{\ln D_{K}}}$ can be best understood in terms of the ramified primes in $K$. These are precisely the primes dividing $D_{K}$. Now, for $S_{K}$ denoting the number of distinct primes dividing $D_{K}$, one can verify

$$
n^{S_{K}} \ll D_{K}^{\frac{C}{\ln \ln D_{K}}},
$$

with equality for $D_{K}$ equaling the product of the first $X$ primes $(X \approx$ $\left.\frac{\ln D_{K}}{\ln \ln D_{K}}\right)$. Thus, for 2-torsion of certain quadratic fields the bound is tight.

- The above methods cannot recover some transfer principles, such as the Scholtz reflection principle which relates 3 -torsion in the quadratic fields $\mathbb{Q}(\sqrt{D})$ and $\mathbb{Q}(\sqrt{-3 D})$, or the work of Ellenberg and Venkatesh [5]. This is because these principles use heavily class field theory, which has not been an input in our work thus far. Nonetheless, one can rephrase and generalize these reflection principles in this language.

\section{Applications to LOWER Bounds for Galois orbits}

In this section we apply results of section 5 to the study Galois orbits of special points in higher rank. Let $A_{g, 1}(\mathbb{C})$ denote the moduli space for principally polarized complex abelian varieties of dimension $g$. We also let $\mathbb{H}_{g}$ denote the Siegel upper half-space, so that

$$
A_{g, 1}(\mathbb{C}) \approx G S p_{2 g}(\mathbb{Q}) \backslash \mathbb{H}_{g} \times G S p_{2 g}\left(\mathbb{A}_{f}\right) / G S p_{2 g}(\hat{\mathbb{Z}}) .
$$

From now on, given a field $F$ we refer to the torus $\operatorname{Res}_{F / \mathbb{Q}} \mathbb{G}_{m}$ simply as $F^{\times}$. 
Let $x$ be a CM point in $A_{g, 1}$ corresponding to a principally polarized abelian variety $A_{x}$. We set $R_{x}$ to be the center of the ring of endomorphisms $\operatorname{End}\left(A_{x}\right)$ of $A_{x}$. All of the CM points in $A_{g, 1}(\mathbb{C})$ are known to be defined over $\overline{\mathbb{Q}}$ and so admit a natural action of the Galois group $\operatorname{Gal}(\overline{\mathbb{Q}} / \mathbb{Q})$. We recall Conjecture 1.1 for the convenience of the reader:

Conjecture 7.1. There exists a positive constant $\delta_{g}$ such that the inequality $|\operatorname{Gal}(\overline{\mathbb{Q}} / \mathbb{Q}) \cdot x| \gg_{g} \operatorname{Disc}\left(R_{x}\right)^{\delta_{g}}$ holds for all $\epsilon>0$ as $x$ varies over all CM points in $A_{g, 1}$.

We begin by reviewing the description of the Galois action on $A$ in terms of the CM theory.

7.1. Background on CM theory. Let $A$ be a principally polarized complex CM abelian variety, which we shall take to be simple for the moment. Then there is a CM field $K$ such that

$$
\operatorname{End}(A) \otimes_{\mathbb{Z}} \mathbb{Q} \cong K
$$

We let $F$ denote the maximal totally real subfield of $K$. Moreover, there is a set $\Sigma_{K}=\left\{\phi_{1}, \phi_{2}, \ldots, \phi_{g}\right\}$ of complex embeddings of $K$ such that the representation of $K$ on the complex tangent space of $A$ is given as the direct sum of the 1-dimensional representations induced by $S$. Moreover, $\Sigma_{K} \cup \sigma_{K}$ give all embeddings of $K$ into $\mathbb{C}$. Let $L$ denote the normal closure of $K$ and $S$ be all the embeddings of $L$ into $\mathbb{C}$ which induce an element of $S$ on $K$. The pair $(K, S)$ is referred to as the CM-type of $A$. Since $A$ is simple, $(K, S)$ must be a primitive CM-type, which means that the right stabilizer of $S_{0}$ in $\operatorname{Gal}(L / \mathbb{Q})$ is $\operatorname{Gal}(L / K)$.

Now, let $M T_{x}^{0}$ denote the torus which is the preimage of the torus $\mathbb{Q}^{\times}$under the norm map on $K$, so that

$$
M T_{x}^{0}:=\operatorname{ker}\left(K^{\times} \rightarrow F^{\times} / \mathbb{Q}^{\times}, a \rightarrow a \bar{a}\right) .
$$

Then corresponding to $A$ there is an embedding of algebraic groups:

$$
\phi_{x}: M T_{x}^{0} \hookrightarrow G S p_{2 g},
$$

and moreover we have the equality

$$
\phi_{x}\left(M T_{x}^{0}(\mathbb{Q})\right) \cap G S p_{2 g}(\hat{\mathbb{Z}}) \approx \operatorname{End}(A)^{0},
$$

where

$$
\operatorname{End}(A)^{0}:=\{a \in \operatorname{End}(A) \mid a \bar{a} \in \mathbb{Z}\} .
$$

Now, there is a reciprocity morphism $r_{x}: L^{\times} \rightarrow M T_{x}^{0}$ given by

$$
r_{x}(a)=\prod_{\tau \in S_{0}} \tau^{-1}(a) .
$$

Since the morphism $r_{x}$ depends only on the CM-type $(K, S)$, we denote it also by $r_{K, S}$. In the future, if there is no confusion, we drop the $S$ and simply write $r_{K}$. We can consider the map

$$
s_{x}:\left(\mathbb{A}_{f}^{L}\right)^{\times} \rightarrow G S p_{2 g}(\mathbb{Q}) \backslash \mathbb{H}_{g} \times G S p_{2 g}\left(\mathbb{A}_{f}\right) / G S p_{2 g}(\hat{\mathbb{Z}})
$$

given by

$$
s_{x}(a) \rightarrow\left(x, \phi_{x} \circ r_{x}(a)\right)
$$

The image of $s_{x}$ is equal to the Galois orbit of the CM point representing $A$. Moreover, there is a unique subtorus $M T_{x} \hookrightarrow M T_{x}^{0}$ such that $r_{x}$ is surjective onto $M T_{x}$ as a morphism of tori, and we call this the Mumford-Tate group of $A$. 
We now review the general theory where $A$ is not necessarily simple. In this case $A$ is isogenous to a product $A_{i}^{n_{i}}$, where each $A_{i}$ is a simple abelian variety with CM such the $A_{i}$ are mutually non-isogenous. Let $\left(K_{i}, S_{i}\right)$ be the CM-type of $A_{i}$ (note that it does NOT follow that the $K_{i}$ are mutually non-isomorphic!). Let $L$ denote the compositum of the normal closures $L_{i}$ of the $K_{i}$, and $S_{i}^{0}$ be the set of embeddings of $L$ into $\mathbb{C}$ which induce one of the $S_{i}$ on $K_{i}$. Let $F_{i}$ denote the maximal totally real subfield of $K_{i}$ and set $M T_{x}^{0}$ to be the preimage of $\mathbb{Q}^{\times}$under the norm map on $\prod_{i} K_{i}$ so that

$$
M T_{x}^{0}:=\operatorname{ker}\left(\prod_{i} K_{i}^{\times} \rightarrow\left(\prod_{i} F_{i}^{\times}\right) / \mathbb{Q}^{\times}, a \rightarrow a \bar{a}\right) .
$$

As before, there is an embedding

$$
\phi_{x}: M T_{x}^{0} \hookrightarrow G S p_{2 g}
$$

and moreover we have the identity

$$
\phi_{x}\left(M T_{x}^{0}(\mathbb{Q})\right) \cap G S p_{2 g}(\hat{\mathbb{Z}}) \approx Z(\operatorname{End}(A))^{0},
$$

where $Z(\operatorname{End}(A))$ is the center of $\operatorname{End}(A)$ and

$$
Z(\operatorname{End}(A))^{0}:=\{a \in Z(\operatorname{End}(A)) \mid a \bar{a} \in \mathbb{Z}\} .
$$

The reciprocity morphism $r_{x}: L^{\times} \rightarrow M T_{x}^{0}$ is now defined to be

$$
r_{x}(a)=\left(\prod_{\tau \in S_{0}^{i}} \tau^{-1}(a)\right)_{i},
$$

where we consider $M T_{x}^{0}$ as a subtorus of $\prod_{i} K_{i}^{\times}$. Again, there is a unique subtorus $M T_{x} \hookrightarrow M T_{x}^{0}$ such that $r_{x}$ is surjective onto $M T_{x}$ as a morphism of tori, and we call this the Mumford-Tate group of $A$. As before, the image of the composition

$$
s_{x}:\left(\mathbb{A}_{f}^{L}\right)^{\times} \rightarrow G S p_{2 g}(\mathbb{Q}) \backslash \mathbb{H}_{g} \times G S p_{2 g}\left(\mathbb{A}_{f}\right) / G S p_{2 g}(\hat{\mathbb{Z}})
$$

is the Galois orbit of the CM point representing $A$.

7.2. Reducing to fields. Consider for a moment Conjecture 7.1 in the case where $A$ is a simple CM abelian variety with $\mathrm{CM}$ by a field $K$ such that the endomorphism ring of $A$ is the full ring of integers $\mathcal{O}_{K}$. Then the map $\phi_{x} \circ r_{x}$ factors through

$$
\widetilde{r_{K}}: C l(L) \rightarrow C l(K) .
$$

A positive answer to Conjecture 7.1 would thus require a positive answer to the following conjecture, which is purely a conjecture about fields:

Conjecture 7.2. Let $K$ be a $C M$ field of degree $2 g$, and let $S$ be a primitive $C M$ type for $K$. Let $L$ be the normal closure of $K$, and let $r_{K}$ denote the reciprocity morphism from $L^{\times}$to $K^{\times}$corresponding to $S$. Then there exists $\delta(g)>0$ such that

$$
\left|\operatorname{im}\left(\widetilde{r_{K}}: C l(L) \rightarrow C l(K)\right)\right| \gg_{g . \epsilon} \operatorname{Disc}(K)^{\delta(g)-\epsilon} .
$$

Our aim in this section is to show that Conjecture 7.2 implies Conjecture 7.1 We then devote the remaining sections to the study of Conjecture 7.2 using the results of sections 4 and 5 .

Theorem 7.1. If Conjecture 7.2 holds for all fields of degree $2 h$, with $h \leq g$, then Conjecture 7.1 holds for $A_{g, 1}$. 
Let $x$ be a CM point representing a principally polarized abelian variety $A$. We use the notation of section 7.1 , so that $A$ is isogenous to $\prod_{i} A_{i}^{n_{i}},\left(K_{i}, S_{i}\right)$ is the CM-type of $A_{i}$, and we set $R_{x}$ to be the center of $\operatorname{End}(A)$. Note that $R$ is in a natural way a subring of $\bigoplus_{i} \mathcal{O}_{K_{i}}$. For ease of notation, we define $\mathcal{O}_{x}=\bigoplus_{i} \mathcal{O}_{K_{i}}$.

Now, let

$$
K_{x}=M T_{x}\left(\mathbb{A}_{f}\right) \cap G S p_{2 g}(\hat{\mathbb{Z}})
$$

so that $K_{x}$ is a finite-index subgroup of the maximal compact subgroup $\operatorname{MT}_{x}(\hat{\mathbb{Z}})$ of $M T_{x}\left(\mathbb{A}_{f}\right)$.

By Yafaev ([17, proposition 2.8), there is a finite index compact subgroup $K_{g}$ of $G S p_{2 g}(\hat{\mathbb{Z}})$ such that if $K_{x}^{\prime}=K_{x} \cap K_{g}$, then the map

$$
M T_{x}(\mathbb{Q}) \backslash M T_{x}\left(\mathbb{A}_{f}\right) / K_{x}^{\prime} \longrightarrow G S p_{2 g}(\mathbb{Q}) \backslash \mathbb{H}_{g} \times G S p_{2 g}\left(\mathbb{A}_{f}\right) / K_{g}
$$

is injective. It follows that

$$
|\operatorname{Gal}(\overline{\mathbb{Q}} / \mathbb{Q}) \cdot x| \gg_{g}\left|\operatorname{im}\left[\left(\mathbb{A}_{L}^{f}\right)^{\times} \rightarrow M T_{x}(\mathbb{Q}) \backslash M T_{x}\left(\mathbb{A}_{f}\right) / K_{x}\right]\right| .
$$

Now, $R_{x}$ is naturally a subring of $\mathcal{O}_{x}$, and

$$
\operatorname{Disc}\left(R_{x}\right)=\left[M T_{x}(\hat{\mathbb{Z}}): K_{x}\right]^{2} \cdot \prod_{i=1}^{m} D_{K_{i}} .
$$

Let $i_{x}$ denote the set of primes $p$ where $p$ divides $\left[\mathcal{O}_{x}: R_{x}\right]$. By Ullmo-Yafaev ([15, lemma 2.13), there is a positive constant $B$ depending only on $g$ such that

$$
\begin{aligned}
\mid \operatorname{im}:\left(\mathbb{A}_{f}^{L}\right)^{\times} \rightarrow & M T_{x}(\mathbb{Q}) \backslash M T_{x}\left(\mathbb{A}_{f}\right) / K_{x} \mid \\
& \gg_{g} B^{\left|i_{x}\right|} \cdot\left[M T_{x}(\hat{\mathbb{Z}}): K_{x}\right] \cdot\left|\operatorname{im}: C l(L) \rightarrow C l\left(M T_{x}\right)\right| .
\end{aligned}
$$

Let us first deal with the second term. There is a surjection

$$
\pi_{i}: M T_{x} \rightarrow M T_{A_{i}},
$$

and we have the equality

$$
\pi_{i} \circ r_{x}=r_{A_{i}} \circ N_{L / L_{i}}
$$

where $N_{L / L_{i}}$ is the norm map from $L$ to $L_{i}$. By class field theory the norm map on class groups has cokernel bounded by the dimension of the fields. Since we are also assuming Conjecture 7.2 , it follows that

$$
\forall i,\left|\mathrm{im}: C l(L) \rightarrow C l\left(M T_{x}\right)\right| \gg_{g} D_{K_{i}}^{\delta\left(g_{i}\right)},
$$

where $g_{i}$ is the dimension of $A_{i}$.

In particular, this proves the theorem unless the index $\left[\mathcal{O}_{x}: R_{x}\right]$ grows faster than any power of the discriminants $D_{K_{i}}$. Thus, the theorem will follow once we prove the following.

Lemma 7.2. There are constants $c_{g}>0, d_{g}$ such that

$$
\left[\mathcal{O}_{x}: R_{x}\right] \leq\left[M T_{x}(\hat{\mathbb{Z}}): K_{x}\right]^{c_{g}} \cdot\left(\prod_{i=1}^{m} D_{K_{i}}\right)^{d_{g}} .
$$

Proof. Since

$$
\left[M T_{x}(\hat{\mathbb{Z}}): K_{x}\right]=\prod_{p}\left[M T_{x}\left(\mathbb{Z}_{p}\right):\left(K_{x}\right)_{p}\right]
$$


and

$$
\left[\mathcal{O}_{x}: R_{x}\right]=\prod_{p}\left[\prod \mathcal{O}_{K_{i}} \otimes_{\mathbb{Z}} \mathbb{Z}_{p}: R_{x} \otimes_{\mathbb{Z}} \mathbb{Z}_{p}\right]
$$

the question is essentially reduced to a local one.

By the proof of Lemma 3.12 in [15], if $p$ is unramified in $L$ and $p \mid\left[\mathcal{O}_{x}: R_{x}\right]$, then

$$
\left[M T_{x}\left(\mathbb{Z}_{p}\right):\left(K_{x}\right)_{p}\right] \gg p .
$$

To finish the proof, we therefore need only to consider the case where $\left[\mathcal{O}_{x}: R_{x}\right]$ is divisible by a large power of some prime $p$.

We must first show that is impossible for $R_{x}$ to decrease without $K_{x}$ also decreasing. To that end, we have the following elementary lemma:

Lemma 7.3. $M T_{x}(\mathbb{Q})$ generates $\bigoplus_{i} K_{i}$ as a ring.

Proof. First, it follows from $S_{i}$ being a primitive CM-type that the only elements of $\operatorname{Gal}\left(L_{i} / \mathbb{Q}\right)$ which fix $r_{A_{i}}(a)$ for all $a \in L_{i}$ are in $\operatorname{Gal}\left(L_{i} / K_{i}\right)$. Thus $M_{x}(\mathbb{Q})$ generates a $\mathbb{Q}$-algebra $E$ in $\bigoplus_{i} K_{i}$ which surjects onto $K_{i}$ for each $i$. Likewise, since the $S_{i}$ are pairwise inequivalent, the only elements of the Galois group of the Galois algebra $\bigoplus_{i} L_{i}$ which fix $E$ are those in the group $\bigoplus_{i} \operatorname{Gal}\left(L_{i} / K_{i}\right)$. The lemma follows.

For a prime $p$ and an element $\alpha \in \mathcal{O}_{L} \otimes_{\mathbb{Z}} \mathbb{Z}_{p}$, define

$$
S(\alpha):=\sum_{\tau \in S} \tau^{-1}(\alpha)
$$

Note that we have the identity

$$
r_{x}\left(e^{p \alpha}\right)=e^{p S(\alpha)} .
$$

We shall need the following more refined version of Lemma 7.3

Lemma 7.4. There is a constant $C$ depending only on $g$ such that we can pick $\alpha \in \mathcal{O}_{L} \otimes_{\mathbb{Z}} \mathbb{Z}_{p}$ with $\mathbb{Z}_{p}[S(\alpha)]$ having index at most $p^{C}$ in $\mathcal{O}_{x}$.

Proof. Let $\psi_{i}$ denote all the ring homomorphisms from $\left(\mathcal{O}_{x}\right)_{p}$ to $\overline{\mathbb{Q}_{p}}$ over $\mathbb{Q}_{p}$. We consider

$$
\psi_{i j}=\psi_{i} \circ S-\psi_{j} \circ S
$$

as a linear map from $L$ to $L$. Then the index of $\mathbb{Z}_{p}[S(\alpha)]$ in $\mathcal{O}_{x}$ is given by $v_{p}(i(\alpha))$, where

$$
i(\alpha)=\prod_{i \neq j} \psi_{i j}(\alpha)
$$

The lemma will follow once we show that there is a uniform constant $C$ and an element $\alpha \in L\left(\mathbb{Z}_{p}\right)$ such that $v_{p}\left(\psi_{i j}(\alpha)\right) \leq p^{C}$. To prove this, recall that if we take a $\mathbb{Z}_{p}$ basis $\beta_{i}$ for $\mathcal{O}_{L} \otimes_{\mathbb{Z}} \mathbb{Z}_{p}$ and let $\left(\sigma_{1}, \ldots, \sigma_{m}\right)$ be the elements of the Galois group of $L_{p}$, then the $p$-valuation of the determinant of the matrix $\left(\sigma_{j}\left(\beta_{i}\right)\right)_{i, j}$ is the discriminant of $L_{p}$ and therefore bounded by $p^{C}$ for some absolute constant $C$. If we replace $\sigma_{1}$ in the matrix by $\psi_{i j}$, then the determinant scales at most by the $p$-power of the coefficient of $\sigma_{1}$ in $\psi_{i j}$, which is uniformly bounded. This finishes the proof.

Lemma 7.5. There is an integer $m$ depending only on $g$ such that if

$$
p^{n} \|\left[M T_{x}\left(\mathbb{Z}_{p}\right):\left(K_{x}\right)_{p}\right],
$$

then $p^{m(n+1)}$ does not divide $\left[\mathcal{O}_{x}: R_{x}\right]$. 
Proof. Pick $\alpha$ as in Lemma 7.4 so that the index of $\mathbb{Z}_{p}[S(\alpha)]$ in $\mathcal{O}_{x}$ is bounded by $p^{C}$. Now set $h$ to be the dimension of $\mathcal{O}_{x}$ and define $\beta_{i}:=e^{i p^{N} S(\alpha)}$ for $0 \leq i \leq h-1$. Note that $\beta_{i} \in K_{x}$. Then

$$
\beta_{i}=1+i p^{N} S(\alpha)+\frac{i^{2} p^{2 N} S(\alpha)^{2}}{2 !}+\cdots+\frac{i^{h-1} p^{N(h-1)} S(\alpha)^{h-1}}{(h-1) !}+p^{N h-h} \cdot\left(\mathcal{O}_{x}\right)_{p} .
$$

The matrix

$$
M_{h}=\left(\frac{i^{j}}{j !}\right)_{0 \leq i, h \leq h-1}
$$

is invertible with some determinant $m_{h}$. Thus, if $N>n+C+m_{h}$, we see that the span of the $\beta_{i}$ contains $p^{N h-h-1} \cdot\left(\left(\mathcal{O}_{x}\right)_{p} / p \cdot\left(\mathcal{O}_{x}\right)_{p}\right)$ and thus $p^{N h-g-1}\left(\mathcal{O}_{x}\right)_{p}$ by Nakayama's lemma. But this means that

$$
\left[\mathcal{O}_{x}: R_{x}\right] \leq p^{N h^{2}-h^{2}-h},
$$

which finishes the proof.

Lemma 7.2 now follows from Lemma 7.5 as discussed above.

7.3. General lower bounds under the Generalized Riemann Hypothesis. Assuming the Generalized Riemann Hypothesis for CM fields, Yafaev [17 proved a lower bound for Galois orbits of special points of the form $c_{N} \log \left(D_{K}\right)^{N}$ for any $N>0$. The idea of his proof was to use small split primes to generate a large number of distinct elements of $C l(K)$. We present here a slight refinement of his method which allows us to get a polynomial lower bound in $D_{K}$, thus showing that GRH implies a positive answer to Conjecture 1.1 in general. Yafaev worked over a general Shimura variety. For simplicity of exposition, we shall only present our method for the case of Siegel space $A_{g, 1}$, as this allows us to work classically, although the method applies just as well for any Shimura variety. The idea is that while Yafaev used very small split primes to generate a large number of class group elements, we use much larger split primes (a small power of the discriminant) to represent lots of distinct elements in the class group.

Theorem 7.6. Assume GRH for CM fields. Then

$$
\left|\operatorname{im}\left(\widetilde{r_{K}}\right)\right| \gg_{g, \epsilon} D_{K}^{\frac{1}{2 g^{2}}-\epsilon}
$$

Proof. Let $\delta$ denote the element of $\operatorname{Gal}(L / \mathbb{Q})$ representing complex conjugation. Consider unramified primes $p, q$ which split completely in $K$, and therefore also in $L$. Choose prime ideals $P, Q$ in $\mathcal{O}_{L}$ above $p, q$ respectively, and suppose that $r_{K}(P)=r_{K}(Q)$ as elements of the ideal class group $C l(K)$. Then there is an element $x \in K$ such that

$$
(x)=\frac{r_{K}(P)}{r_{K}(Q)}
$$

and hence

$$
\left(x / x^{\delta}\right)=\frac{r_{K}(P) r_{K}\left(Q^{\delta}\right)}{r_{K}(Q) r_{K}\left(P^{\delta}\right)} .
$$

Since $K$ is a primitive CM-type and $P$ and $Q$ are totally split, $\frac{x}{x^{\delta}}$ must generate $K$ over $\mathbb{Q}$. Now, consider the element

$$
y=\frac{p q x}{x^{\delta}} .
$$


Then $y$ is an algebraic integer which generates $K$ over $\mathbb{Q}$. Moreover, all conjugates of $y$ are of absolute value $p q$. Since $\operatorname{Disc}(\mathbb{Z}[y]) \geq D_{K}$ we arrive at the inequality

$$
p q \gg_{g} D_{K}^{\frac{1}{g^{2}}}
$$

It follows that

$$
\left|\operatorname{im}\left(\widetilde{r_{K}}\right)\right|>\#\left\{p \mid p \text { splits completely in } K \text { and } p \ll_{g} D_{K}^{\frac{1}{2 g^{2}}}\right\} .
$$

Since GRH implies the existence of at least $D_{K}^{\frac{1}{2 g^{2}}-\epsilon}$ such primes, we are done.

7.4. Setting up the combinatorics. For each $g$, define $W_{g}$ to be the Weyl group of order $g$, which we will view as a group of signed permutations. That is, an element of $W_{g}$ is a permutation $\sigma$ in $S_{g}$ together with a choice, for each $i$, of sign $\sigma_{i}$. If $\sigma(i)=j$, then we say that $\sigma$ takes $i$ to $j$ with sign $\sigma_{i} \in\{+1,-1\}$. We define composition by multiplying signs. That is, $(\sigma \circ \tau)(i)=\sigma(\tau(i))$ and $(\sigma \circ \tau)_{i}=\sigma_{\tau(i)} \cdot \tau_{i}$. It is convenient to use the following notation for an element of $W_{g}$ : the element $\sigma=\left(1^{+} 3^{-}\right)\left(2^{-}\right)$is used to denote the permutation $(13)(2)$ which takes 1 to 3 with positive sign, 3 to 1 with negative sign and 2 to itself with negative sign. So for instance, $\sigma^{2}=\left(1^{-}\right)\left(3^{-}\right)\left(2^{+}\right)$. Note that there is a map $W_{g} \rightarrow S_{g}$ taking a signed permutation to the underlying permutation, and this map is a surjective homomorphism. The kernel of this map will be denoted by $W_{g}^{0}$. Thus $W_{g}^{0}$ is a normal subgroup of $W_{g}$ isomorphic to $(\mathbb{Z} / 2 \mathbb{Z})^{g}$.

As before, let $(K, S)$ denote a CM field of degree $g$ together with a primitive CM-type $\Sigma_{K}=\left(\phi_{1}, \ldots, \phi_{g}\right)$. Let $L$ be the Galois closure of $K$, and $G$ be the Galois group $\operatorname{Gal}(L / \mathbb{Q})$. Let $S \subset \operatorname{Gal}(L / \mathbb{Q})$ denote all elements inducing an element of $\Sigma_{K}$ on $K$. We also let $\delta \in G$ be complex conjugation. Then $G$ can canonically be viewed as a subgroup of $W_{g}$ in the following way: For each $g \in G$ and $1 \leq i \leq g$, we have that $g\left(\phi_{i}\right)$ is either $\phi_{j}$ for some $j$, or $\overline{\phi_{j}}$ for some $j$. Thus, we can define $\sigma_{g}$ to be the signed permutation mapping $i$ to $j$ with sign 1 if $g\left(\phi_{i}\right)=\phi_{j}$ and $\operatorname{sign}-1$ if $g\left(\phi_{i}\right)=\overline{\phi_{j}}$. It is easy to see that this is an injective homomorphism $\operatorname{Gal}(L / \mathbb{Q}) \hookrightarrow W_{g}$. It is worthwhile noting under this map that complex conjugation always maps to the element $\left(1^{-}\right)\left(2^{-}\right) \ldots\left(g^{-}\right)$. We assume wlog that $\phi_{1}$ is the identity embedding on $K$. It follows under this identification that $S$ consists of the set of all elements of $G$ that send the element 1 to some $j$ with sign 1 , and thus $S^{-1}$ is the set of all elements which send some $i$ to 1 with sign 1 . Define $H$ to be $\operatorname{Gal}(L / K)$, so that $H$ is the subgroup of $G$ which sends 1 to itself with sign 1 . Since we assumed that the CM-type $\Sigma_{K}$ is primitive, the right stabilizer of $S$ in $G$ must be $H$.

7.5. Understanding the map $r_{K}$ on characters and cocharacters. Let us begin by understanding the relevant $G$-modules. A basis for $X\left(K^{\times}\right)$is the set of all embeddings $\psi_{i}: K \rightarrow \mathbb{C}$, where the Galois action is given by composition: $g\left(\psi_{j}\right)=g \circ \psi_{j}$. Likewise, a basis for $X\left(L^{\times}\right)$is the set of all embeddings $\xi_{j}: L \rightarrow$ $\mathbb{C}$, where the Galois action is again given by composition. By fixing an identity embedding $L \hookrightarrow \mathbb{C}$ we can consider the elements $g$ of $G$ as a basis for $X\left(L^{\times}\right)$. The map $\widehat{r_{K}}$ sends $\psi_{i}$ to

$$
\widehat{r_{K}}\left(\psi_{i}\right)=\psi_{i} \circ\left(\prod_{g \in S^{-1}} g\right),
$$


where we now consider $g$ as an element of $X\left(L^{\times}\right)$. It will be more natural to consider the map on cocharacters for us. We take the dual bases $g^{*}$ for $X\left(L^{\times}\right)^{*}$ and $\psi_{i}^{*}$ for $X\left(K^{\times}\right)^{*}$. The dual map ${\widehat{r_{K}}}^{*}$ takes an element $g^{*}$ to

$$
{\widehat{r_{K}}}^{*}\left(g^{*}\right)=\prod_{1 \leq i \leq g} \delta^{\frac{\left(1-\sigma_{i}\right)}{2}}\left(\phi_{i}^{*}\right),
$$

where $\sigma$ is the element of $W_{g}$ corresponding to $g^{-1}$. That is, $\phi_{i}^{*}$ appears in the sum if and only if $g$ takes some element $j$ to $i$ with sign 1 .

Denote by $L_{0}$ the kernel of the norm map $N_{L / \mathbb{Q}}: L^{\times} \rightarrow \mathbb{Q}^{\times}$and define $K_{0}$ to be the kernel of the norm map from $K$ to its totally real subfield $F$ given by

$$
N_{K / F}(k)=k \bar{k} \text {. }
$$

$X\left(L_{0}\right)$ is the quotient of $X\left(L^{\times}\right)$by the primitive vector $\sum_{g \in G} g$ and $X\left(L_{0}\right)^{*}$ is the submodule of $X\left(L^{\times}\right)^{*}$ consisting of all elements $\sum_{g \in G} a_{g} \cdot g^{*}$ such that $\sum_{g} a_{g}=$ 0. Similarly, $X\left(K_{0}\right)$ is the quotient of $X\left(K^{\times}\right)$by the relations $\phi_{i}=-\bar{\phi}_{i}$ and $X\left(K_{0}\right)^{*}$ is spanned by the elements $\pi_{i}^{*}:=\phi_{i}^{*}-\bar{\phi}_{i}^{*}, 1 \leq i \leq g$. Note that $r_{K}$ takes $L_{0}$ to $K_{0}$.

Define $M$ to be the image of $X\left(K_{0}\right)$ in $X\left(L_{0}\right)$ by $\widehat{r_{K}}$ and let $T_{M}$ be the torus whose character lattice is $M$.

Lemma 7.7. We have $D_{T_{M}} \gg_{g} D_{K}^{\frac{1}{2 g-1}}$.

Proof. Since $x$ corresponds to a simple abelian variety, the CM-type $(K, S)$ must be primitive and so the stabilizer of the element $\sum_{g \in S^{-1}}(g-\bar{g})$ in $G$ is $H$. Note that this element lies in $M$. Since $L$ is the Galois closure of $K$ we know that $H$ contains no non-trivial normal subgroup of $G$, so we see that the action of $G$ on $M$ has trivial kernel. The result then follows by considering the Artin conductor of the associated representation and applying Theorem 4.7 and Lemma 4.6.

Note that $T_{M}(\mathbb{R})$ is compact, so $T_{M}$ has trivial regulator. In particular, by Theorem 5.1 we can conclude that the Galois orbit of $x$ is large as long as $M$ is a primitive sublattice of $X\left(L_{0}\right)$. This is equivalent to the image of $X\left(L_{0}\right)^{*}$ in $X\left(K_{0}\right)^{*}$ being primitive. This is the description we shall work with.

7.6. Lower bounds for Galois orbits of Weyl CM fields. Following Chai and Oort 2, we say that $K$ is a Weyl CM field if the $\operatorname{Galois} \operatorname{group} \operatorname{Gal}(L / \mathbb{Q})$ is isomorphic to $W_{g}$. Weyl CM fields can be thought of as the generic CM fields, much like a generic field of degree $g$ has Galois group $S_{g}$. The following lemma combined with Theorem 7.1 proves Theorem 1.2

Lemma 7.8. If $K$ is a Weyl CM field, then we have

$$
\left|\operatorname{im}\left(\widetilde{r_{K}}\right)\right| \gg_{g, \epsilon} D_{K}^{\frac{1}{4}-\epsilon} .
$$

Proof. Since $K_{0}$ fits into an exact sequence of tori

$$
F^{\times} \rightarrow K^{\times} \rightarrow K_{0}
$$

and the Artin conductor is multiplicative, it follows from Corollary 4.5 that

$$
D_{K_{0}} \gg_{\epsilon} \frac{D_{K}^{1-\epsilon}}{D_{F}} \geq D_{K}^{1 / 2}
$$

so the lemma will follow once we show that ${\widehat{r_{K}}}^{*}$ maps $X\left(L_{0}\right)^{*}$ surjectively onto $X\left(K_{0}\right)^{*}$. 
For $1 \leq i \leq g$, let $g_{i} \in \operatorname{Gal}(L / \mathbb{Q})$ be the element such that

$$
g_{i}\left(\phi_{j}\right)= \begin{cases}\phi_{j} & i \neq j, \\ \bar{\phi}_{i} & i=j .\end{cases}
$$

Note that we know $g_{i}$ exists precisely because $K$ is a Weyl CM field. Now, $1^{*}-g_{i}^{*}$ is in $X\left(L_{0}\right)^{*}$ and maps onto $\pi_{i}^{*}$ in $X\left(K_{0}\right)^{*}$. Since the $\pi_{i}^{*} \operatorname{span} X\left(K_{0}\right)^{*}$, this completes the proof.

7.7. Proofs for $g=2,3,4,5$, and 6. In this subsection we prove that Galois orbits are large for $g=2,3,4,5,6$. We record one final combinatorial fact before proceeding:

Lemma 7.9. For each $i \neq 1$ and $\epsilon \in\{+1,-1\}$ there exists an element $g$ in $S$ which takes $i$ somewhere with sign $\epsilon$.

Proof. Suppose for the sake of a contradiction that there is an $i \neq 1$ and an $\epsilon \in$ $\{+1,-1\}$ such that $\forall g \in S, g$ takes $i$ to $g(i)$ with sign $\epsilon$. Since $G$ acts transitively on the set $\{1,2, \ldots, g\}$ there is a $g \in G$ with $g(1)=i$. Multiplying $g$ by $\delta$ if necessary, we can also ensure that $g$ takes 1 to $i$ with sign $\epsilon$. But then $g$ is in the right stabilizer of $S$ and $g \notin H$, contradicting the fact that $S$ induces a primitive CM-type on $K$.

The following theorem combined with Theorem 7.1 proves Theorem 1.1

Theorem 7.10. Conjecture 7.2 holds for $g=2,3,4,5$ with

and

$$
\begin{gathered}
\delta(2)=\delta(3)=\frac{1}{4}, \\
\delta(4)=\frac{1}{7}, \\
\delta(5)=\frac{1}{9},
\end{gathered}
$$

$$
\delta(6)=\frac{1}{12}
$$

Remark. While $\delta(2)$ and $\delta(3)$ are optimal, we have made no attempts to optimize $\delta(4), \delta(5)$ or $\delta(6)$. Since the proof reduces $\delta(4)$ to a discriminant calculation, optimizing $\delta(4)$ is a straightforward exercise in representation theory, while optimizing $\delta(5)$ would involve bounding 3-torsion in class groups of quadratic fields. Of course, optimizing the values $\delta_{g}$ for Conjecture 7.1 would require more work and, in particular, a better way to handle the case of a non-maximal endomorphism ring.

Proof. The theorem follows from Lemmas 7.11, 7.12, 7.13, 7.14, 7.15 and the previous Lemma 7.7 .

Lemma 7.11. For $g=2$, the image of ${\widehat{r_{K}}}^{*}$ is all of $X\left(K_{0}\right)^{*}$.

Proof. $X\left(K_{0}\right)^{*}$ is generated by $\pi_{1}^{*}$ and $\pi_{2}^{*}$. We write $(a, b)$ to denote the element $a \pi_{1}^{*}+b \pi_{2}^{*}$. Recall that $X\left(L_{0}\right)^{*}$ is generated by the elements $g^{*}-1^{*}$.

Now, we have

$$
{\widehat{r_{K}}}^{*}\left(\delta^{*}-1^{*}\right)=(-1,-1) .
$$

Moreover, by Lemma 7.9 there is an element in $S$ which sends 1 somewhere with sign 1 while sending 2 somewhere with sign -1 . This must either be $\left(1^{+} 2^{-}\right)$or 
$\left(1^{+}\right)\left(2^{-}\right)$. So either $(0,-1)$ or $(-1,0)$ is in the image of ${\widehat{r_{K}}}^{*}$. This together with $(-1,-1)$ already generates, so we're done.

Lemma 7.12. For $g=3$, the image of ${\widehat{r_{K}}}^{*}$ is all of $X\left(K_{0}\right)^{*}$.

Proof. Similarly to the case $g=2$, we write $(a, b, c)$ to denote $a \pi_{1}^{*}+b \pi_{2}^{*}+c \pi_{3}^{*}$. We have

$$
{\widehat{r_{K}}}^{*}\left(\delta^{*}-1^{*}\right)=(-1,-1,-1) .
$$

Since $G$ acts transitively on the embeddings $\phi_{1}, \phi_{2}, \phi_{3}$, it must contain a 3-cycle. Suppose $G$ has a 3 -cycle $\sigma$, where not all the signs are the same. By multiplying $\sigma$ with complex conjugation $\delta$ we can ensure that two of the signs are positive. Assume $\sigma=\left(1^{+} 2^{+} 3^{-}\right)$. Then

$$
{\widehat{r_{K}}}^{*}\left(1^{*}-\sigma^{*}\right)=(1,0,0)
$$

and

$$
{\widehat{r_{K}}}^{*}\left(1^{*}-\left(\sigma^{2}\right)^{*}\right)={\widehat{r_{K}}}^{*}\left(1^{*}-\left(1^{+} 3^{-} 2^{-}\right)^{*}\right)=(1,1,0)
$$

and these 2 vectors combined with $(-1,-1,-1)$ generate $X\left(K_{0}\right)^{*}$.

The same logic holds for the other such cycles. Thus, we are reduced to the case where $G$ only contains 3 -cycles where all signs are the same. Since $G$ contains $\delta$ we see that $G$ must contain all 3 -cycles where all signs are the same, in particular the element $\sigma=\left(1^{+} 2^{+} 3^{+}\right)$.

Recall that the image of ${\widehat{r_{K}}}^{*}$ is closed under the action of $G$. By Lemma 7.9, $G$ contains an element $\tau$ which takes something to 1 with a positive sign and something to 2 with a negative sign. Hence, ${\widehat{r_{K}}}^{*}\left(1^{*}-\tau^{*}\right)$ is either $(0,1,0)$ or $(0,1,1)$. Now, acting by $\sigma$ we see that the image of $\widehat{r}_{K}{ }^{*}$ contains either all of $(0,1,0),(0,0,1),(1,0,0)$ or $(0,1,1),(1,0,1),(1,1,0)$. Either way, together with $(1,1,1)$ we see that this generates $X\left(K_{0}\right)^{*}$.

Lemma 7.13. For $g=4$, the size of the image of $\widetilde{r_{K}}$ is at least $c_{\epsilon} D_{K}^{\frac{1}{7}-\epsilon}$.

Proof. Again, we use the notation $(a, b, c, d)$ to denote $a \pi_{1}^{*}+b \pi_{2}^{*}+c \pi_{3}^{*}+d \pi_{4}^{*}$. Let

$$
N_{K}:=\{(a, b, c, d) \mid a+b+c+d \equiv 0 \bmod 2\} .
$$

Now, as $X\left(L_{0}\right)^{*}$ is generated by the elements $1^{*}-g^{*}$, we see that the image of $\widehat{r}_{K}^{*}$ is the span of some subset $W$ of the vectors

$$
\left\{\left(a_{1}, a_{2}, a_{3}, a_{4}\right) \mid a_{i} \in\{0,1\}, 1 \leq i \leq 4\right\}
$$

which includes the vector $v=(1,1,1,1)$. We claim that the image of $\widehat{r}_{K}^{*}$ is either primitive or the sublattice $N_{K}$.

To see this, note first that by replacing $w$ by $v-w$ we can assume all vectors in $W$ with 1 or 2 entries to be 1 , and the remaining entries to be 0 . Now, if $W$ contains a vector with a single entry being 1 , then since the image of ${\widehat{r_{K}}}^{*}$ is $G$-invariant it must contain all the basis vectors and thus be the whole thing. We can thus assume that $W$ only contains vectors with exactly 2 entries being 1 . These come in pairs:

$$
\{(1,1,0,0),(0,0,1,1)\},\{(1,0,1,0),(0,1,0,1)\},\{(1,0,0,1),(0,1,1,0)\} .
$$

If $S$ contains 1 or 2 such pairs, then the span can easily be checked to be primitive. Hence the only case where the image is not a primitive sublattice is if $S$ contains all 3 such pairs, and the image is thus $N_{K}$.

Now, if the image is primitive, the lemma follows by Lemma 7.7. We thus restrict to the case where the image is $N_{K}$. Let $T_{K}$ be the torus whose cocharacter 
module is $N_{K}$, and consider the induced map $T_{M} \rightarrow K_{0}$. It is sufficient to show that the cokernel of the induced map on class groups is discriminant negligible. But $X\left(L_{0}\right)^{*} / N_{K} \approx \mathbb{Z} / 2 \mathbb{Z}$, and so it must be a trivial Galois module. The result therefore follows from Corollary 5.2 .

Lemma 7.14. For $g=5$, the size of the image of $\widetilde{r_{K}}$ is at least $c_{\epsilon} D_{K}^{\frac{1}{9}-\epsilon}$.

Proof. We use the notation $(a, b, c, d, e)$ to denote $a \pi_{1}^{*}+b \pi_{2}^{*}+c \pi_{3}^{*}+d \pi_{4}^{*}+e \pi_{5}^{*}$. Now, if the span of $\widehat{r}_{K}^{*}$ is primitive, we're done by Lemma 7.7, so we assume from now on that this is not the case. As $X\left(L_{0}\right)^{*}$ is generated by the elements $1^{*}-g^{*}$, we see that the image of ${\widehat{r_{K}}}^{*}$ is the span of some subset $W$ of the vectors

$$
\left\{\left(a_{1}, a_{2}, a_{3}, a_{4}, a_{5}\right) \mid a_{i} \in\{0,1\}, 1 \leq i \leq 5\right\}
$$

which includes the vector $v=(1,1,1,1,1)$. By replacing $w$ by $v-w$ we can assume that all vectors in $W$ except $v$ have 1 or 2 entries that are 1 , and at least 3 entries that are 0 . If any vectors in $W$ have exactly 1 component that is 1 , then this eliminates a variable and reduces us to the $g=4$ case above, so we can assume all vectors in $W$ have exactly 2 entries that are 1 . Thus the image contains a vector $\pi_{z}^{*}+\pi_{w}^{*}$ for some $z \neq w$. Now, note that the image of ${\widehat{r_{K}}}^{*}$ is closed under the action of $G$. Moreover, since $G$ contains an element of order 5 , it must contain a 5 -cycle. Taking a power of this 5-cycle we can conclude that $G$ contains a 5 -cycle $\sigma$ which takes $z$ to $w$ (and has some signs). Acting on the vector $\pi_{i}^{*}+\pi_{j}^{*}$ by powers of $\sigma$ we see that the cokernel $B$ is generated by $\pi_{z}^{*}$ with each $\pi_{k}^{*}$ equaling $\pm \pi_{z}^{*}$. We also have the relation $\pi_{1}^{*}+\pi_{2}^{*}+\pi_{3}^{*}+\pi_{4}^{*}+\pi_{5}^{*}=0$ in $B$. Since $\pi_{w}^{*}=-\pi_{z}^{*}$, we can conclude that either $B$ is trivial or $B \approx \mathbb{Z} / 3 \mathbb{Z}$. Since we assumed that $B$ isn't trivial, we must have $B \approx \mathbb{Z} / 3 \mathbb{Z}$.

Our goal is now to bound $|C k(B)|$. The automorphism group of $B$ as an abelian group is $\mathbb{Z} / 2 \mathbb{Z}$, so we get a map from $\operatorname{Gal}(L / \mathbb{Q})$ to $\mathbb{Z} / 2 \mathbb{Z}$. The kernel $G_{1}$ must be of index 2. Now consider the quadratic field $k$ fixed by $G_{1}$. Notice that $k \subset K$ since $\operatorname{Gal}(L / K)$ fixes $\pi_{1}^{*}$ and thus acts trivially on $B$. Now, the module $X\left(k^{\times}\right)^{*} / 2 X\left(k^{\times}\right)^{*}$ is an extension of $B$ by a trivial Galois module, so by Corollary 5.2 we have that $|C k(B)|=|C l(k)[2]|$ up to discriminant negligible factors. In particular,

$$
|C k(B)| \leq|C l(k)| \leq D_{k}^{\frac{1}{2}}
$$

The rest is easy: since $k \subset K$, we have $D_{k}^{5} \leq D_{K}$. Also, $D_{K_{0}} \gg_{\epsilon} D_{K}^{1-\epsilon} / D_{F}$, where $F$ is the maximal totally real subfield of $K$, so $D_{F}^{2} \leq D_{K}$. Thus

$$
D_{K_{0}} \gg_{\epsilon} D_{K}^{-\epsilon} \cdot \frac{D_{K}^{\frac{1}{5}}}{D_{k}^{\frac{1}{2}}} \geq D_{K}^{\frac{3}{20}-\epsilon}>D_{K}^{\frac{1}{9}},
$$

as desired.

Lemma 7.15. For $g=6$, the size of the image of ${\widetilde{r_{K}}}^{*}$ is at least $c_{\epsilon} D_{K}^{\frac{1}{12}-\epsilon}$.

Proof. Let $B$ be the cokernel of $\widehat{r}_{K}^{*}$. As $X\left(L_{0}\right)^{*}$ is generated by the elements $1^{*}-g^{*}$, we see that the image $U$ of ${\widehat{r_{K}}}^{*}$ is the span of some subset $W$ of the vectors

$$
\left\{\sum_{i=1}^{6} a_{i} \pi_{i}^{*} \mid a_{i} \in\{0,1\}, 1 \leq i \leq 6\right\}
$$

which includes the vector $v=(1,1,1,1,1,1)$. Given a vector $w=\sum_{i=1}^{6} c_{i} \pi_{i}^{*}$, we refer to $c_{i}$ as the $i$ th entry of $w$. By replacing $w$ by $v-w$ we can assume that all 
vectors in $W$ have 1,2 or 3 entries that are 1 , and at least 3 entries that are 0 . Recall that $U$ is invariant by $G$. Since $G$ acts transitively on the vectors $\pm \pi_{i}^{*}$, we deduce that the order of the $\pi_{i}^{*}$ (possibly infinite) in $B$ is independent of $i$. If $B$ is torsion-free, then the lemma follows from Lemma 7.7. so we assume from now on that this isn't the case.

Lemma 7.16. The image of ${\widehat{r_{K}}}^{*}$ contains an element of the form $\pi_{i}^{*} \pm \pi_{j}^{*}$, for $j \neq i$.

Proof. If $W$ contains a vector with exactly 1 entry being 1 , then for some $i$ we have $\pi_{i}^{*}=0$ in $B$, and hence by the $G$-invariance of $U$ we see that $B$ must be trivial, contradicting our assumption that $B$ is torsion-free. If $W$ contains an element with exactly 2 entries being 1 's, then we're done. Hence the only remaining case is where all the vectors in $W$ have exactly 3 entries being 1 's. Let $v_{1}$ be one such vector. If $W$ consists only of $v_{1}$ and/or $v-v_{1}$, then it is easy to see that $B$ is isomorphic to $\mathbb{Z}^{4}$ and hence torsion free. So $W$ must contain at least 2 vectors $v_{1}, v_{2}$ with 3 non-zero entries and $v_{1} \notin\left\{v_{2}, v-v_{1}\right\}$. Then either $v_{1}$ and $v_{2}$ or $v_{1}$ and $v-v_{2}$ have two of the same entries being 1 . Assume wlog that $v_{1}$ and $v_{2}$ have two of the same entries being 1 . Then $v_{1}-v_{2}$ has one entry equal to 1 , one entry equal to -1 , and the rest equal to 0 . This proves the lemma.

By acting by $G$ on the vector produced by Lemma 7.16 we deduce that $U$ contains an element of the form $\pi_{1}^{*} \pm \pi_{j}^{*}$, and by relabeling we can assume it contains $\pi_{1}^{*} \pm \pi_{2}^{*}$. Now, $W_{6}$ acts naturally on $X\left(L_{0}\right)^{*}$ and $U$ is invariant by $G$. Since $U$ contains $\pi_{1}^{*} \pm \pi_{2}^{*}$, we see that $U$ is also invariant either by $\left(1^{+} 2^{+}\right)$or $\left(1^{-} 2^{-}\right)$. Let $G^{U}$ denote the subgroup of $W$ preserving $U$, so that $G^{U}$ contains $G$ and this signed transposition. Let $G^{0}$ denote the image of $G^{U}$ in $S_{6}$, so that $G^{0}$ is a transitive subgroup of $S_{6}$ containing (12). Draw a graph $V_{U}$ on $\{1,2,3,4,5,6\}$, where two elements $i, j$ are connected by an edge if $(i j) \in G^{0}$. Since $(i j)$ and $(j k)$ generate $(i k)$, we see that this graph is a disjoint union of complete graphs. Since $G^{0}$ acts on this graph transitively, the complete graphs must have the same size. We thus see that either $V_{U}$ is the complete graph, in which case $G^{0}$ is either all of $S_{6}$, or $V_{U}$ is 2 triangles or 3 disjoint edges. We thus split into 3 cases:

- Case i: $G^{0} \approx S_{6}$.

The $G^{U}$ orbit of $\pi_{1}^{*} \pm \pi_{2}^{*}$ thus contains either $\pi_{1}^{*}+\pi_{j}^{*}$ or $\pi_{1}^{*}-\pi_{j}^{*}$ for each $j \neq 1$. Thus $B$ is cyclic and generated by $\pi_{1}^{*}$. Since we assumed that $B$ is torsion, we see that $U$ has full rank in $X\left(L_{0}\right)^{*}$. Now, each element of $G$ takes $\pi_{1}^{*}$ to $\pm \pi_{j}^{*}$ for some $j$. As $\pi_{j}^{*}= \pm \pi_{1}^{*}$ in $B$, we see that the $G$ action on $B$ factors through $\mathbb{Z} / 2 \mathbb{Z}$. Taking the kernel of the action of $G$ on $B$, we see that there is a quadratic field $k \in K$ such that $|C k(B)| \leq|C l(k)|$. But $|C l(k)| \ll_{\epsilon} D_{k}^{\frac{1}{2}+\epsilon} \ll D_{K}^{\frac{1}{12}+\epsilon}$. Thus,

$$
\left|\operatorname{im}\left(\widetilde{r_{K}}\right)\right| \gg_{\epsilon} \frac{D_{K}^{\frac{1}{2}-\epsilon}}{D_{F}^{\frac{1}{2}} D_{k}^{\frac{1}{2}}} \gg D_{K}^{\frac{1}{6}-\epsilon} .
$$

- Case ii: $V_{U}$ consists of 2 triangles with vertices $\{1,2,3\}$ and $\{4,5,6\}$. In this case $\left\{\{1,2,3\},\{4,5,6\}\right.$ must be a $G^{0}$-quotient set.

As in Case i, we have that in $B$,

$$
\pi_{1}^{*}= \pm \pi_{2}^{*}= \pm \pi_{3}^{*}, \quad \pi_{4}^{*}= \pm \pi_{5}^{*}= \pm \pi_{6}^{*} .
$$


Let $G_{0}$ denote the stabilizer of $\pi_{1}^{*}$ in $G$. Then $G_{0}$ contains $H$ as a subgroup of index at most 3 , so that there is a field $k \in K$ of degree at most 4 over $\mathbb{Q}$ such that $|C k(B)| \leq|C l(k)|$. But $|C l(k)| \ll_{\epsilon} D_{k}^{\frac{1}{2}+\epsilon} \ll D_{K}^{\frac{1}{6}+\epsilon}$. Thus,

$$
\left|\operatorname{im}\left(\widetilde{r_{K}}\right)\right| \gg_{\epsilon} \frac{D_{K}^{\frac{1}{2}-\epsilon}}{D_{F}^{\frac{1}{2}} D_{k}^{\frac{1}{2}}} \gg D_{K}^{\frac{1}{12}-\epsilon} .
$$

- Case iii: $V_{U}$ consists of 3 disjoint edges $\{1,2\},\{3,4\}$ and $\{5,6\}$.

In this case $\{\{1,2\},\{3,4\},\{5,6\}\}$ must be a $G^{0}$-quotient set. Also, we have

$$
\pi_{1}^{*}= \pm \pi_{2}^{*}, \quad \pi_{3}^{*}= \pm \pi_{4}^{*}, \quad \pi_{5}^{*}= \pm \pi_{6}^{*}
$$

in $B$.

Suppose that $\pi_{1}^{*}$ is 2-torsion in $B$. Then the stabilizer $G_{0} \subset G$ of $\pi_{1}^{*}$ in $B$ has $H$ as a subgroup of index at least 4 , and so there is a subfield $k \subset K$ of degree at most 3 such that

$$
|C k(B)| \leq|C l(k)| \ll_{\epsilon} D_{k}^{\frac{1}{4}+\epsilon} .
$$

Thus

$$
\left|i m\left(\widetilde{r_{K}}\right)\right| \gg_{\epsilon} \frac{D_{K}^{\frac{1}{2}-\epsilon}}{D_{F}^{\frac{1}{2}} D_{k}^{\frac{1}{2}}} \gg D_{K}^{\frac{1}{8}-\epsilon} .
$$

Henceforth we assume that $\pi_{i}^{*}$ is not 2-torsion in $B$.

Consider a vector $w \in W$. If $w$ has 1 non-zero entry, then $B$ is trivial as before and we're done. If $w$ has 2 non-zero entries, then they must both be $\{1,2\},\{3,4\}$ or $\{5,6\}$ by the main assumption of this case. If $W$ consists only of some subset of

$$
\left\{\pi_{1}^{*}+\pi_{2}^{*}, \pi_{3}^{*}+\pi_{4}^{*}, \pi_{5}^{*}+\pi_{6}^{*}\right\},
$$

then since $U$ is generated by $W$ and $v$ and $W \cup v$ can be completed to a basis of $X\left(L_{0}\right)^{*}$, it follows that $B$ is torsion-free. Hence we can assume $W$ contains a vector $w$ with 3 non-zero coefficients.

Thus, $w \in W$ is of the form $w=\pi_{i}^{*}+\pi_{j}^{*}+\pi_{k}^{*}$. Suppose $i, j$ both belong to the same set $\{1,2\},\{3,4\}$ or $\{5,6\}$. Wlog $i=1, j=2, k=3$. Then we see that either $B$ is trivial contrary to assumption, or $\pi_{3}^{*}=-2 \pi_{1}^{*}$ in $B$. Now, the action of $G^{0}$ on the 3 -element set $\{\{1,2\},\{3,4\},\{5,6\}\}$ is transitive, so it must contain both 3 -cycles. Acting on the relation $\pi_{3}^{*}=-2 \pi_{1}^{*}$ in $B$ by $G^{U}$ thus gives $\pi_{5}^{*}= \pm 2 \pi_{3}^{*}$ in $B$ and $\pi_{1}^{*}= \pm 2 \pi_{5}^{*}$, and so $\pi_{1}^{*}= \pm 8 \pi_{1}^{*}$ in $B$. Hence $B$ is cyclic, generated by $\pi_{1}^{*}$, and $\pi_{1}^{*}$ has order 3,7 or 9 . But $v-w=\pi_{4}^{*}+\pi_{5}^{*}+\pi_{6}^{*}$ is in $U$, which gives the relation $\pi_{4}^{*}=-2 \pi_{5}^{*}= \pm \pi_{1}^{*}$ in $B$, so that $\pi_{1}^{*}= \pm 2 \pi_{1}^{*}$ and $\pi_{1}^{*}$ must have order 3 in $B$. We can now proceed as in case $\mathrm{i}$.

Hence we can assume that any $w$ with 3 non-zero coefficients must be of the form $w=\pi_{i}^{*}+\pi_{j}^{*}+\pi_{k}^{*}$ with each of $\{i, j, k\}$ belonging to a different element of $\{\{1,2\},\{3,4\},\{5,6\}\}$. Recalling the relations $\pi_{1}^{*}= \pm \pi_{2}^{*}, \pi_{3}^{*}=$ $\pm \pi_{4}^{*}$ and $\pi_{5}^{*}= \pm \pi_{6}^{*}$ in $B$, we see that each such $w$ imposes a relation in $B$ of the form $\pi_{1}^{*} \pm \pi_{3}^{*} \pm \pi_{5}^{*}=0$. Any 2 distinct such relations imply that $2 \pi_{i}^{*}=0$ in $B$ for some $i$, which we assumed was not the case. Hence $B$ 
is isomorphic as an abelian group to the free module on $\pi_{1}^{*}, \pi_{3}^{*}, \pi_{5}^{*}$ modded out by a single relation $\pi_{1}^{*} \pm \pi_{3}^{*} \pm \pi_{5}^{*}=0$. Hence $B$ is torsion-free, which is a contradiction.

\section{ACKNOWLEDGEMENTS}

It is with great pleasure that the author thanks Peter Sarnak for introducing him to this problem, and he gratefully thanks the referee for reading the paper carefully and helping to substantially improve the exposition.

\section{REFERENCES}

[1] A. Brumer and J. H. Silverman, The number of elliptic curves over $\mathbb{Q}$ with conductor $N$, Manuscripta Math. 91 (1996), no. 1, pp. 95-102. MR.1404420 (97e:11062)

[2] C. Chai and F. Oort, Abelian Varieties Isogenous to a Jacobian, http://www. math. upenn. edu/ chai/papers_ pdf/isogJac65. pdf, preprint, 2010

[3] B. Edixhoven, On the André-Oort conjecture for Hilbert modular surfaces, Moduli of abelian varieties (Texel Island, 1999), Progr. Math. 195, Birkhäuser, Basel, 2001, pp. 133-155. MR.1827018 (2002c:14042)

[4] B. Edixhoven, B. Moonen and F. Oort, Open problems in algebraic geometry, Bull. Sci. Math., vol. 125. (2001), pp. 1-22. MR1812812(2002a:14001)

[5] J. Ellenberg and A. Venkatesh, Reflection principles and bounds for class group torsion, Int. Math. Res. Not. 2007, no. 1. MR2331900 (2008f:11124)

[6] F. Gerth, Ranks of 3-Class Groups of non-Galois Cubic Fields, Acta Arithmetica, XXX, 1976, pp. 307-322. MR0422198(54:10190)

[7] B. Klingler and A. Yafaev, The André-Oort conjecture, http://www. math. jussieu. fr/ klingler/papiers/KY12. pdf, preprint, 2008.

[8] T. Nakayama, Cohomology of class field theory and tensor product modules, Ann. of Math. (2), 65 (1957), pp. 255-267. MR0090620(19:841b)

[9] T. Ono, On the Tamagawa Number of Algebraic Tori, Annals of Mathematics, Vol. 78, No. 1, July, 1963. MR0156851(28:94)

[10] T. Ono, Arithmetic of Algebraic Tori, Annals of Mathematics, Vol. 74, No. 1, July, 1961. MR 0124326(23:A1640)

[11] J. Pila, O-minimality and the André-Oort conjecture for $\mathbb{C}^{n}$, http://www.maths.ox.ac. uk/system/files/PilaAOMML4. pdf, to appear in Annals of Math.

[12] V. Platonov and A. Rapinchuk, Algebraic Groups and Number Theory, Academic Press, 1993. ISBN0125581807 MR1278263 (95b:11039)

[13] G. Shimura, On Abelian Varieties with Complex Multiplication, Princeton Mathematical Series 46, Princeton University Press, 1998. MR 1492449 (99e:11076)

[14] J. Shyr, On Some Class Number Relations of Algebraic Tori, Michigan Math Journal, Vol. 24, Issue 3, 1977, pp. 365-377. MR0491596 (58:10819)

[15] E. Ullmo and A. Yafaev, Galois orbits and equidistribution of special subvarieties : towards the André-Oort conjecture. http://www.math.u-psud.fr/ ullmo/Prebublications/ UllmoYafaev2(3)(2)(2).pdf

[16] E. Ullmo and A. Yafaev, Nombre de classes des tores de multiplication complexe et bornes inférieures pour orbites Galoisiennes de points spéciaux. preprint.

[17] A. Yafaev, A conjecture of Yves André's, Duke Mathematical Journal, Vol. 132, No. 3, 2006, pp. 393-408. MR2219262(2007b:11089)

[18] S. Zhang, Equidistribution of CM points on Quaternionic Shimura Varieties, International Mathematics Research Notices, No. 59, 2005, pp. 3657-3689. MR2200081 (2007g:11067)

Department of Mathematics, Princeton University, Fine Hall, Washington Road, Princeton, New Jersey 08544-1000

Current address: Department of Mathematics, Faculty of Arts \& Sciences, Harvard University,

One Oxford Street, Cambridge MA 02138

E-mail address: jacobt@math.harvard.edu 